\title{
Review of observations of ground diffusion in space and in time and fractal model of ground motion
}

\author{
Vladimir Shiltsev \\ Fermi National Accelerator Laboratory, P.O. Box 500, Batavia, Illinois 60510, USA
}

(Received 8 June 2010; published 9 September 2010)

\begin{abstract}
We present numerous observations of the diffusive motion of the ground and tunnels for scientific instruments and show that if systematic movements are excluded the remaining uncorrelated component of the motion obeys a characteristic fractal law with the displacement variance $d Y^{2}$ scaling with time and spatial intervals $T$ and $L$ as $d Y^{2} \propto T^{\alpha} L^{\gamma}$ with both exponents close to 1 ( $\alpha \approx \gamma \approx 1$ ). We briefly describe experimental methods of the mesoscopic and microscopic ground motion detection used in measurements at physics research facilities sensitive to ground motion, particularly large high energy elementary particle accelerators. A simple mathematical model of the fractal motion demonstrating the observed scaling law is also presented and discussed. This paper is a subsequent full detail publication to [V. Shiltsev, Phys. Rev. Lett. 104, 238501 (2010)].
\end{abstract}

DOI: 10.1103/PhysRevSTAB.13.094801

PACS numbers: 29.20.db, 89.75.Da, 05.45.Df, 91.10.Kg

\section{INTRODUCTION}

Motion of ground was always of practical interest because of the threat of earthquake-induced damage and concerns about structural stability of buildings due to large movements. In recent decades, development of large-scale facilities for scientific research also confronted the issue of very tight tolerances on the position of individual elements in the presence of microscopic motion of the ground. The most notable examples are gravitational wave detectors [13] and high energy particle accelerators [4-7]. For gravitational wave detectors, the ground vibrations transferred to the motion of the mirrors in the arms of interferometers are one of the sources of noise limiting minimum detectable strain. For accelerators, motion of numerous focusing magnets disturbs the trajectories of charged particle beams with their small cross sections, affecting machine performance. Given the tight tolerances on positioning, quite sophisticated measurement, stabilization, and correction/ alignment systems are routinely employed in accelerators [8]. To design such systems, one relies on certain phenomenological models of the ground motion which should predict the expected displacement of the ground $\boldsymbol{Y}(t, s)$ in time and space. The spatial scales of interest $L$ for these physics instruments range from several meters to dozens of $\mathrm{km}$ and the time intervals of interest $T$ range from milliseconds to years.

The instruments for studying microscopic ground motion were originally developed for geophysics research. Now many of these are easily applicable for other purposes and have been commercialized. Among those widely used at the large physics facilities are optical interferometers, stretched wires and hydrostatic level systems (HLS) [9], laser position trackers [10], and geophones [11]. They are quite capable of detecting movements over the scales of $L$ and $T$ discussed above even under very quiet conditions.
Ambient ground motion has three distinct components-periodic motion (for example, due to Earth tides, seasonal changes, etc.), systematic drifts or trends (e.g., due to temperature or air pressure variations, precipitation history, etc.), and stochastic movements [12]. The stochastic component usually is less correlated in space, less persistent in time, and less predictable than the first two while not necessarily smaller in amplitude. Thus, the stochastic component often poses the biggest concern. Space-, time-, or space-time variograms can be used to describe average characteristics of the motion $\boldsymbol{Y}(t, s)$ :

$$
\begin{aligned}
\left\langle d Y^{2}(t, L)\right\rangle= & \left\langle(Y(t, s+L)-Y(t, s))^{2}\right\rangle \\
\left\langle d Y^{2}(T, L)\right\rangle= & \langle[Y(t+T, s+L)-Y(t+T, s) \\
& \left.-Y(t+T, s)+Y(t, s)]^{2}\right\rangle,
\end{aligned}
$$

where the brackets $\langle\cdots\rangle$ denote averaging over continuous or discrete time series and $T$ and $L$ are the lags in time and in space, respectively. In this article we present and discuss evidences that the stochastic component of the ground motion can be described as diffusion both in time and in space and has a characteristic fractal law variogram:

$$
\left\langle d Y^{2}(T, L)\right\rangle \propto T^{\alpha} L^{\gamma}
$$

with both exponents close to $1(\alpha \approx \gamma \approx 1)$ over wide ranges of time and space intervals. The corresponding power spectral density (PSD) $P(\omega, k)$ in frequency $\omega=$ $2 \pi f$ and spatial wave number $k=2 \pi / \lambda$ for such a process scales as

$$
P(\omega, k) \propto \frac{1}{\omega^{\beta} k^{\delta}}
$$

with exponents $\beta=\alpha+1$ and $\delta=\gamma+1$. (Mathematical methods of the geophysical time series analysis are discussed in detail in [12].) 
Power-law scaling of separately calculated temporal and spatial variograms of the ground motion, i.e., the dependencies such as $\left\langle d Y^{2}(T, L=\right.$ const $\left.)\right\rangle \propto T^{\alpha}$ and $\left\langle d Y^{2}(t=\right.$ const, $L)>\propto L^{\gamma}$, have long been known to geophysicists (see, e.g., [13,14]). However, it was in high precision studies of numerous dynamics measurement points for large accelerators where simultaneous space and time diffusion was observed for the first time. An empirical $A T L$ law [15] was proposed to summarize the experimental data, according to which the mean square of relative displacement $d Y^{2}$ of the points separated by distance $L$ grows with the time interval between measurements $T$ as

$$
\left\langle d Y^{2}\right\rangle=A T L,
$$

where $A$ is a site dependent constant of the order of $10^{-5 \pm 1} \mu \mathrm{m}^{2} /(\mathrm{s} \mathrm{m})$. Such a wandering of the ground elements takes place in all directions. Because the diffusive coefficient $A$ is small, the diffusive motion presents only a small contribution to the ground motion. For example, in the time period of $1 \mathrm{~h}$ the amplitude of the absolute surface motion (i.e., measured by a seismometer) could be as big as $100 \mu \mathrm{m}$, while the ATL law gives an estimate of the relative displacement of about $1 \mu \mathrm{m}$ for the points $30 \mathrm{~m}$ apart. One would not worry about this contribution except it describes very important, at least for accelerators, uncorrelated background on top of the larger amplitude ground movements correlated in time and space. Some of the most notable examples of such correlated movements are low-frequency seismic waves, the Earth tides, the ambient low-frequency ground motion caused by winds and air pressure variations, temperature gradients, ground water dynamics, precipitation, etc.

Obviously, the ATL law is a particular case of the more general equation (1). The PSDs of the ATL-type motion in the frequency and the wave-number domains scale as

$$
\begin{aligned}
P(f) & =\frac{A L}{2 \pi^{2} f^{2}}, & f>0 \quad \text { and } \\
P(k) & =\frac{2 A T}{k^{2}}, & k>0 .
\end{aligned}
$$

This article reviews the evidences of space-time diffusion of ground surfaces or in underground tunnels. In Sec. II, we discuss the measurements made at particle accelerators with the use of geodetic survey and alignment instruments, describe briefly the impact of misalignments on the beams in accelerators, and present evidence of the beam orbit diffusion caused by diffusion of elements' positions. Section II also reviews the results of various geophysical studies made either at accelerator facilities, or at the sites of future accelerators, or at the geophysics laboratories. We summarize all the measurements and discuss the limits of validity of the space-time ground diffusion laws in Sec. III and present a simple physical model of the fractal ground motion which demonstrates the landscape evolution according to the empirical law.

\section{GROUND AND BEAM ORBIT DIFFUSION IN ACCELERATORS}

\section{A. Impact of ground motion on operation of accelerators}

For the purposes of this study, particle accelerators can be considered as a sequence of linear focusing elements (magnetic lenses) arranged either in a circle (circular accelerators) or in a line (linear accelerators). In an ideal accelerator with perfectly aligned magnetic elements, the beam orbit passes through the centers of the lenses. Any alignment error results in beam orbit distortion. If the distortions are large compared to either the apertures of the lenses or the size of the vacuum chambers or the size of a linear focusing field areas, then they get in the way of reliable machine operation and must be corrected-either by using electromagnetic orbit correctors or by mechanical realignment which brings the centers of the focusing lenses back to their ideal positions [16]. In large accelerators, such as the $6.3-\mathrm{km}$ circumference proton-antiproton Tevatron Collider (Fermilab, Batavia, IL, USA), the 27$\mathrm{km}$ circumference proton-proton Large Hadron Collider (LHC, at CERN, Switzerland), the $6.3 \mathrm{~km}$ circumference proton-electron collider HERA at DESY (Hamburg, Germany), and 25-50 km long future electron-positron linear colliders, all of which have many hundreds of magnetic elements, the ground motion is the most serious cause of the beam orbit distortions. It has to be noted that the biggest effect is produced by uncorrelated relative motion of the neighboring focusing elements while very longwavelength movements are practically unimportant, and, for example, accelerators are not sensitive to their global displacements as a whole [6,7]. Orbit distortions from numerous uncorrelated sources add in quadrature and, thus, the mean squared distortion of the beam orbit due to the ATL-law type ground motion (4) in a circular accelerator with circumference $C$ can be approximated as [17]

$$
\left\langle d Y_{\text {orbit }}^{2}\right\rangle \approx \kappa A T C .
$$

Not surprisingly, Eq. (6) predicts larger orbit drifts in larger circumference accelerators. The numerical factor in (6) $\kappa \approx 2-5$ depends on the design of the beam focusing optics. Typically, the ground motion effects start to be of a serious concern for accelerators at amplitudes of the uncorrelated motion from a fraction of a micron to dozens of microns, depending on the accelerator parameters and types. For example, for accelerators which collide particle beams with very small cross sections, the tolerances on the final focusing magnet position stability could be as tight as a few nanometers to a few microns [7]. Because of such concerns, large accelerators have been usually constructed in deep concrete-and-steel enforced tunnels with typical diameters/sizes of the order of 5-8 m, at 10-100 m depths in the locations with known good and stable geology. 


\section{B. Orbit drifts in large accelerators}

To a greater or lesser extent, long-term orbit drifts are seen at all accelerators. Typically machine operators or/and automatic correction systems counteract the drifts. As large colliding beam facilities are particularly sensitive to orbit motion, several extended studies of the issue have been carried out at them. In this section we present observations of the beam orbit drifts in several large accelerators-HERA (Germany), TRISTAN (Japan), the Tevatron (U.S.), and LEP (Switzerland). Detailed parameters of these machines can be found in the corresponding references below.

\section{Orbit drifts in HERA proton-electron collider}

HERA is a high energy accelerator in Hamburg (Germany), which was in operation as a proton-electron collider from 1992 to 2007. The circumference of HERA is $6.3 \mathrm{~km}$. The facility is located in an underground tunnel at approximately 25 meters depth. It did consist of two independent accelerator storage rings-one for $30 \mathrm{GeV}$ electrons and another for $820 \mathrm{GeV}$ (since 1998-920 GeV) protons-installed in the same tunnel. The height difference between the electron and the proton beam was $0.8 \mathrm{~m}$. The focusing optics lattices of these rings were quite different.

Figure 1 from [18] shows the mean square of the HERA electron ring vertical orbit drifts accumulated after various time intervals (up to 1 month) and detected by 288 beam position monitors equally placed some $23 \mathrm{~m}$ from each other all over the machine circumference. One can see that the variance of the distortions increases approximately

\section{mean square orbit drift}

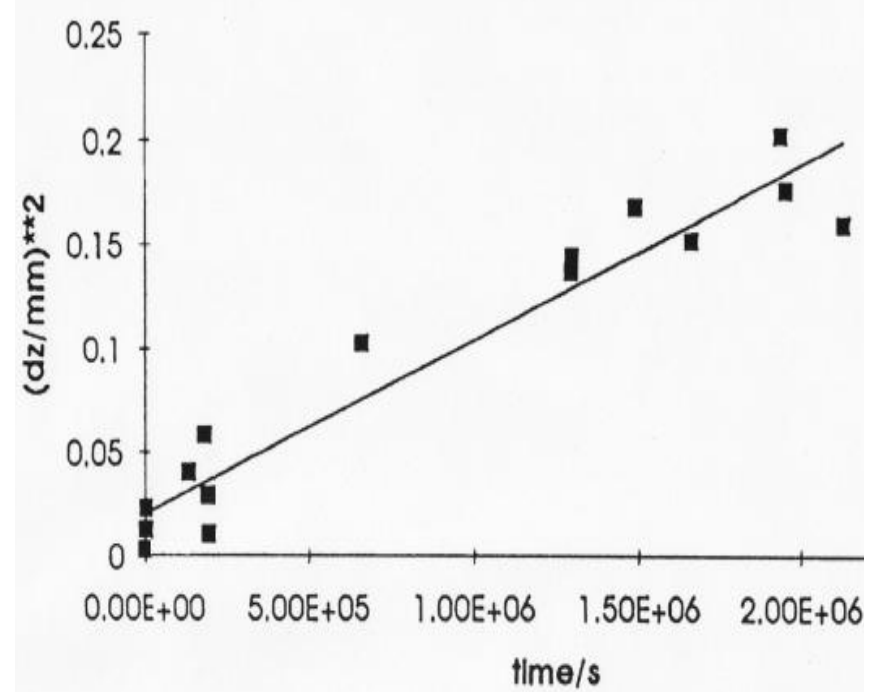

FIG. 1. (Color) Mean square difference of vertical orbit distortions in the HERA electron ring vs time interval duration obtained from data stored during 1993 operation data [18]. linearly in time $\left\langle d Y_{\text {orbit }}{ }^{2}(T)\right\rangle=a+b T$ with $a=$ $0.02 \mathrm{~mm}^{2}$ and $b=8 \times 10^{-8} \mathrm{~mm}^{2} / \mathrm{s}$. Here, the constant $a$ accounts for the measurement's error, while the slope $b$ gives an estimate of the diffusive ground motion constant $A_{\text {HERAe }} \approx 4 \times 10^{-6} \mu \mathrm{m}^{2} / \mathrm{s} / \mathrm{m}$ if one uses the optics coefficient $\kappa \approx 3.1$ for HERA-e [see Eq. (5)].

Analysis of the vertical motion in the proton ring is summarized in the power spectral density (PSD) shown in Fig. 2. The squares at lower frequencies represent the Fourier spectra of the proton orbit differences from different running periods of the accelerator [18]. The procedure was to measure the closed orbit position at all 131 BPMs in the HERA-proton machine and subtract the result from a previous one to obtain the difference orbit, indicating any eventual orbit drift. The analysis of difference orbits was limited to time intervals of a maximum of 5 days during which no intentional change of the closed orbit occurred (i.e., the orbits were left uncorrected). The continuous line represents the Fourier spectrum of readings from one specific beam position monitor in the accelerator [17]. As continuous observations were performed repetitively within several hours of the proton beam lifetime, the lowest frequency of this spectrum is about $0.5 \mathrm{mHz}$. Series of peaks in the spectrum above $1 \mathrm{~Hz}$ are due to cultural seismic noise which is quite prominent in a big city like Hamburg. The dashed line in Fig. 2 shows the PSD scaling $P_{\text {orbit }}(f)=8 \times 10^{-4}\left[\mu \mathrm{m}^{2} \mathrm{~s}\right] / f^{2}$ as expected from the ATL law with the constant $A_{\text {HERAp }} \approx$ $8 \times 10^{-6} \mu \mathrm{m}^{2} / \mathrm{s} / \mathrm{m}$ which fits very well the data in the range of frequencies from $2 \times 10^{-6} \mathrm{~Hz}$ to about $2 \times$ $10^{-2} \mathrm{~Hz}$. In the time domain such a PSD corresponds to irregular noisy "random walk"-like proton orbit drifts over time intervals from a few minutes to several days. Motion of the focusing magnets was found to be the cause of the HERA orbit drifts, as other sources-long-term drifts of

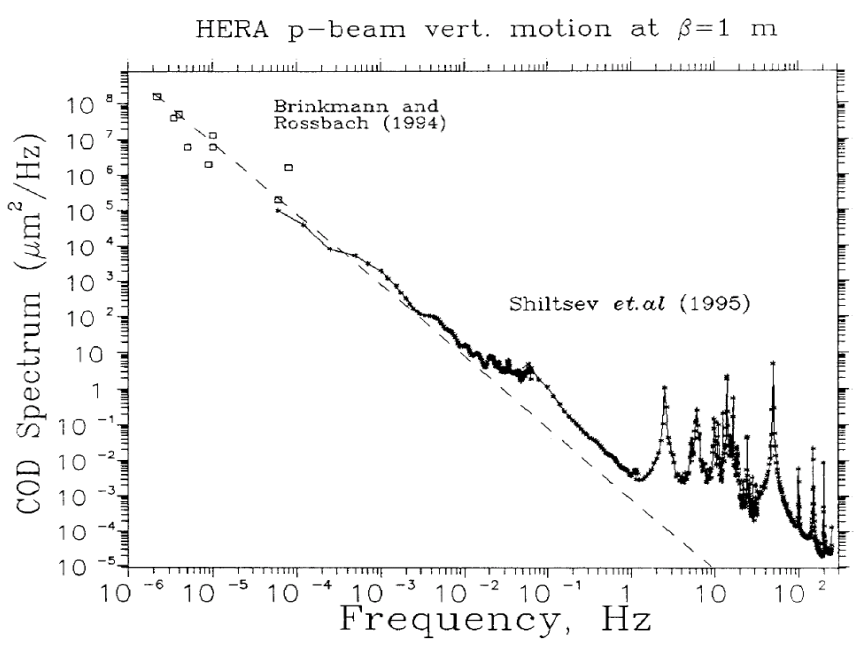

FIG. 2. PSD of the HERA-proton orbit vertical motion normalized to a specific location of the ring. The dashed line corresponds to the ATL law $[17,18]$. 
orbit corrector strengths and low-frequency noises of the BPMs-were negligible.

\section{Orbit drifts in TRISTAN and KEK-B positron-electron colliders}

TRISTAN is a high energy accelerator in Tsukuba (Japan), which was in operation as positron-electron collider in 1986-1998. Its tunnel has about $3.0 \mathrm{~km}$ circumference, has $0.8 \mathrm{~m}$ thick concrete walls, and is set at a depth of approximately 12 meters below the surface. The energies of the beams of positrons and electrons were up to $32 \mathrm{GeV}$. Long-term $8 \mathrm{GeV}$ beam orbit drifts over several periods of a few days each have been reported in Ref. [19] and are shown in Fig. 3. Full circles in the figure are the rms values of the beam positions $x_{i}$ in all $N=392$ BPMs while the open circles represent the rms of the position changes during operation cycles between successive corrections of the orbit, i.e., $\sigma=\left[\Sigma\left(x_{i}-x_{i 0}\right)^{2} / N\right]^{1 / 2}$.

Note that the horizontal closed orbit distortion (COD) is smaller than the vertical one. At large orbit distortions, the beam current circulating in the accelerator degraded sig-

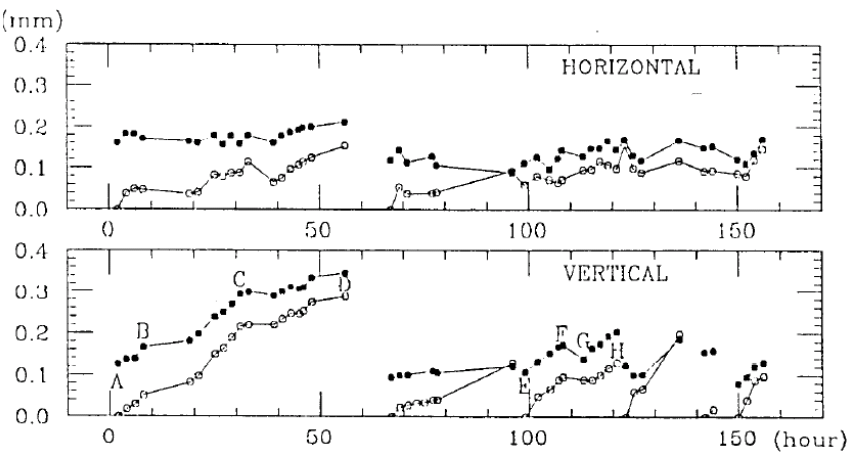

FIG. 3. Changes of rms vertical and horizontal orbits in TRISTAN ring (from [19]).

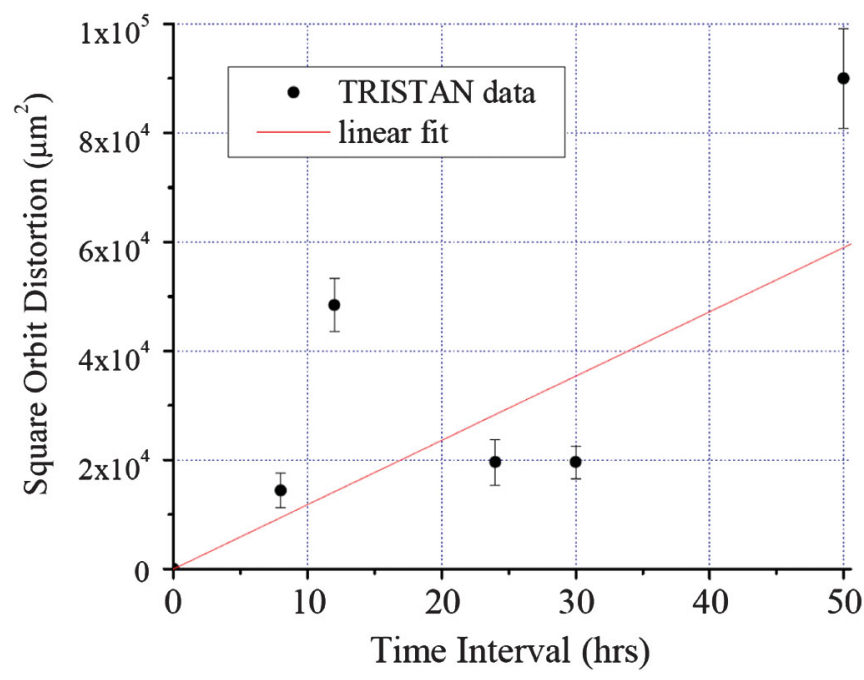

FIG. 4. (Color) Variance of the TRISTAN orbit variations [17]. nificantly so that a correction of the orbit was needed toward the "ideal" orbit (sharp drops at points D, E, H, and some others in Fig. 3).

Analysis of the data presented in Fig. 3 shows that the variance of the COD grows with the time [17]— see Fig. 4-and can be approximated by a linear fit (6) with coefficient $A_{\text {TRISTAN }}=(27 \pm 7) \times 10^{-6} \mu \mathrm{m}^{2} / \mathrm{s} / \mathrm{m}$.

After the end of the TRISTAN operation, it was replaced by a higher performance KEK-B positron-electron collider ("B-factory") that was built in the same tunnel and started its operation in 1999. The KEK-B collider consists of two intersecting rings set side by side-one for $8 \mathrm{GeV}$ electrons and another for $3.5 \mathrm{GeV}$ positrons. Tight sub-mm control of the ring's 3-km circumference is critical for the collider operation. Figure 5 shows a 4 month record of the positron ring circumference change [20].

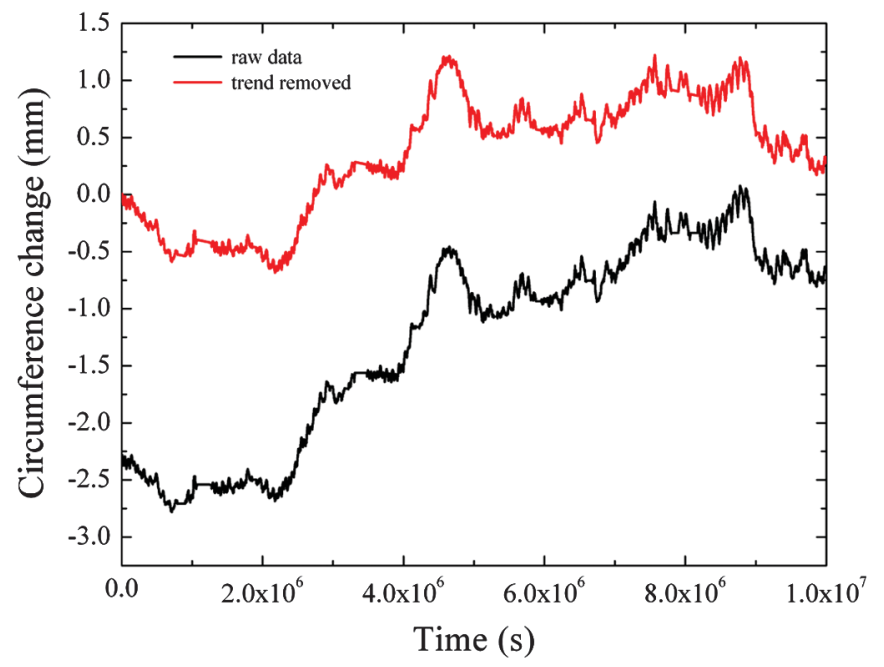

FIG. 5. (Color) KEK-B circumference variations from March 1 to June 30, 2002 [20] (data courtesy of Katsunobu Oide of KEK).

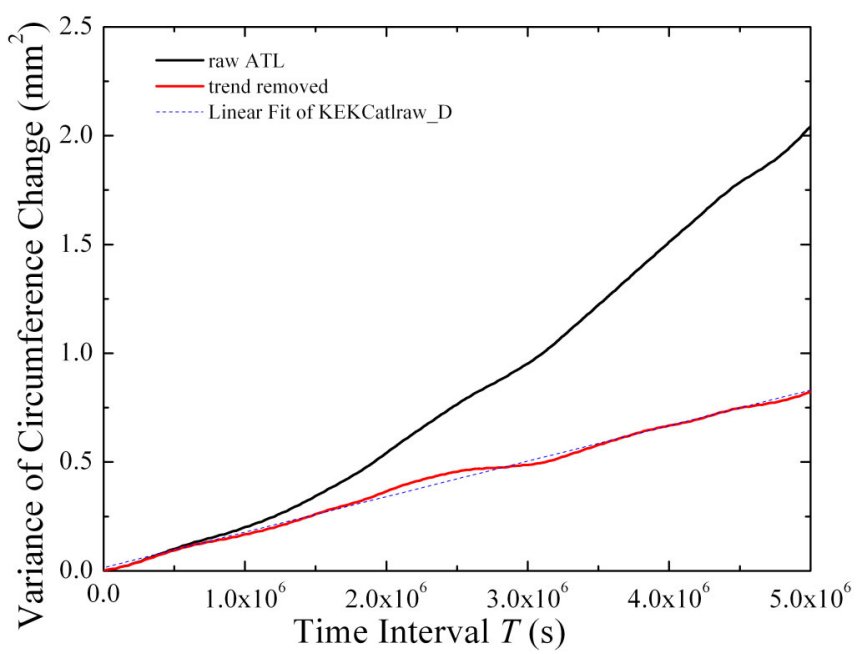

FIG. 6. (Color) Variance of the KEK-B circumference variations; the black line is for raw data, the red line is for the data with linear trend subtracted, and the dashed line is a linear fit. 


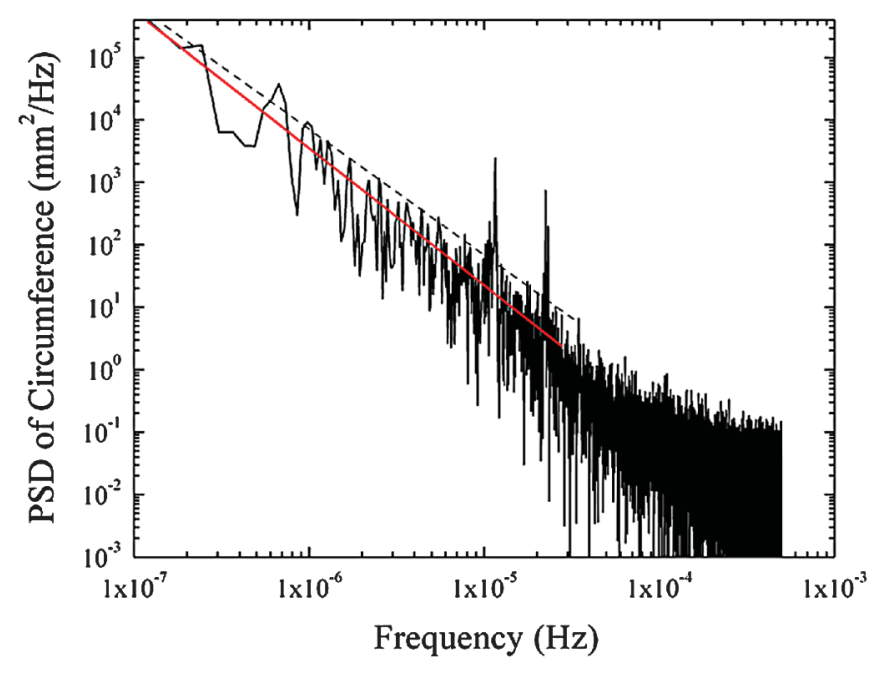

FIG. 7. (Color) Spectrum of the KEK-B circumference variations [21]. The dashed line is for the ATL-law scaling $8.2 / f^{2}$; the solid red line is for a power-law fit $(1.74 \pm 0.2) / f^{2.21 \pm 0.07}$.

If the linear trend is excluded from the data (see upper curve in Fig. 5) then the variogram Eq. (1) of the circumference change $\Delta C$ after a time interval $T$ scales linearly with $T$ - see Fig. 6-as expected from the ATL law $\left\langle\Delta C^{2}\right\rangle=2 A T C$ with $A_{\mathrm{KEK}}=(27 \pm 3) \times$ $10^{-6} \mu \mathrm{m}^{2} / \mathrm{s} / \mathrm{m}$-in good agreement with the TRISTAN orbit drift analysis results presented above.

The PSD of the KEK-B circumference change is presented in Fig. 7 and shows distinctive peaks at frequencies of $\sim 2$ /day (some $15 \mu \mathrm{m}$ changes caused by the tunnel expansion due to solar and lunar tides) and some $30 \mu \mathrm{m}$ peak due to daily temperature changes. The circumference also found changing due to air pressure variations, which were especially prominent during the time when a typhoon hit the area (not in the Fig. 3 data). At very low frequencies below $10^{-5} \mathrm{~Hz}$, the PSD scales approximately as $1 / f^{2.2 \pm 0.1}$, which is in a decent agreement with Eq. (5).

\section{Orbit drifts in Tevatron Proton-Antiproton collider}

Until very recently (2009), the Tevatron Collider has been the world's highest energy accelerator for high energy physics research. It employs $980 \mathrm{GeV}$ beams of protons and antiprotons circulating in opposite directions inside the same set of 774 bending magnets and 216 focusing magnets. The collider is located at Fermi National Accelerator Laboratory (Batavia, Illinois, USA) in a $6.3 \mathrm{~km}$ circumference tunnel at approximately $7 \mathrm{~m}$ below the surface. The motion of the tunnel floor translates into motion of focusing magnets and results in beam orbit distortions. For effective operation of the collider, the beam orbit motion must be stabilized to within $0.1 \mathrm{~mm}$ by means of the automatic orbit correction system. Without such a system, the daily changes are as big as $0.2-0.3 \mathrm{~mm}$ as indicated in Fig. 8 and can reach $0.5-1 \mathrm{~mm}$ over the periods of $2-$ 4 weeks [21].

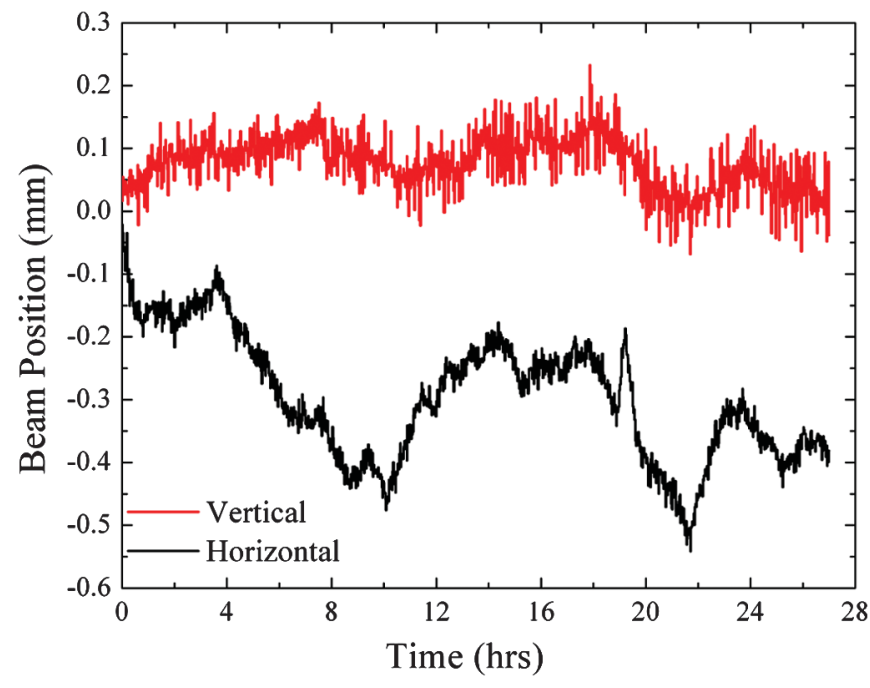

FIG. 8. (Color) Horizontal and vertical orbit motion as measured by one of the beam position monitors in the Tevatron [21].

Besides the 12- and 24-hour variations associated with the tides and daily temperature effects, the orbit motion has a diffusive component. To separate the latter, one can compute the variance of the second differences $\left\langle d d Y^{2}(T)\right\rangle$ which is equal to

$$
\left\langle d d Y^{2}(T)\right\rangle=\left\langle[d Y(t)-2 d Y(t+T)+d Y(t+2 T)]^{2}\right\rangle .
$$

It is easy to see that contrary to variance of the (first) difference (1), the second difference effectively filters out linear trends and slow periodic variations. Indeed, for the process which contains a linear trend, a periodic component, a diffusive ATL-like component, and truly uncorrelated noise (e.g., due to measurement errors) $d Y(t)>=E t+F \sin (\omega t)+($ ATL-like diffision $)+$ (noise with rms of $G$ ), one gets

$$
\left\langle d Y^{2}(T)\right\rangle=E^{2} T^{2}+2 F^{2} \sin ^{2}(\omega T / 2)+\mathrm{ATL}+2 G
$$

$$
\left\langle d d Y^{2}(T)\right\rangle=8 F^{2} \sin ^{4}(\omega T / 2)+2 \mathrm{ATL}+6 G .
$$

Figure 9 shows the result of such analysis for the Tevatron orbit drift data. One can see that both horizontal and vertical variances have significant diurnal (tide) components. The ATL-diffusion components scale linearly with the time lag $T$ and are indicated by dashed lines which have slopes of $0.0027 \pm 0.0003 \mathrm{~mm}^{2}$ over 12 hours (horizontal) and $006 \pm 0.001 \mathrm{~mm}^{2}$ over 12 hours (vertical). The diffusive coefficient $A$ can be calculated from (6) and (8b) taking into account that beam optics factors $\kappa$ are different for horizontal and vertical planes [21], so $A_{\text {Tevatron } V}=(2.6 \pm 0.3) \times 10^{-6} \mu \mathrm{m}^{2} / \mathrm{s} / \mathrm{m}$ and $A_{\text {Tevatron } H}=(1.8 \pm 0.2) \times 10^{-6} \mu \mathrm{m}^{2} / \mathrm{s} / \mathrm{m}$. 


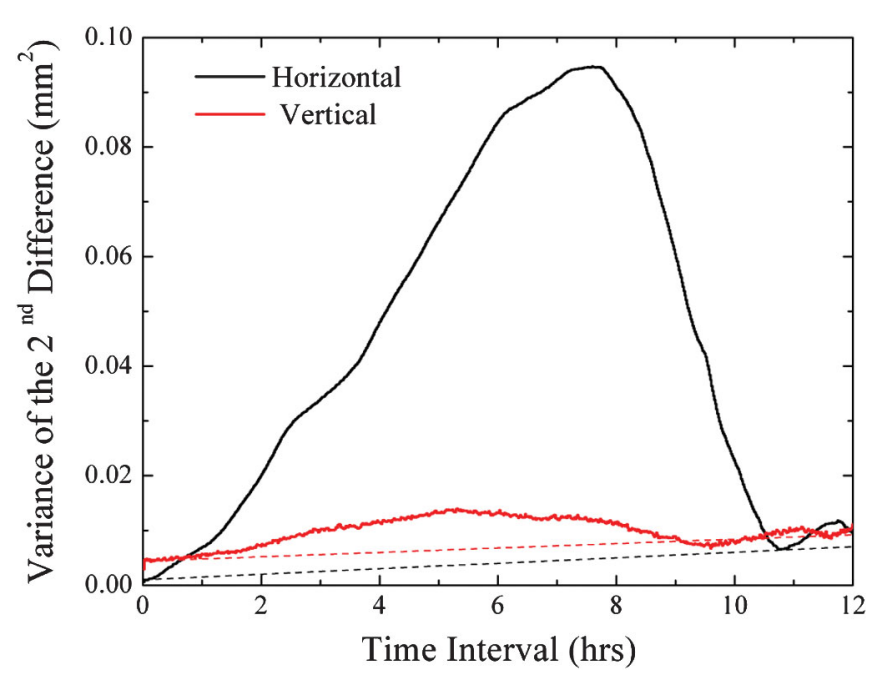

FIG. 9. (Color) Tevatron proton orbit 2nd difference variance. Dashed lines are linear fits of the ATL-like component of the variance.

\section{Orbit drifts in CERN's Large Electron-Positron collider (LEP) and Super-Proton Synchrotron (SPS)}

The large electron-positron collider (LEP) was the world's highest energy electron-positron collider under operation in European Organization for Nuclear Research (CERN) in Geneva, Switzerland in 1989-2000. The maximum beam energy reached $104 \mathrm{GeV} .3368$ bending magnets of LEP deflected the particles and kept them in orbit. There were also 816 focusing magnets and 700 orbit correctors. The $26.7 \mathrm{~km}$ circumference tunnel had eight straight sections and eight arcs and lied between 45 and $170 \mathrm{~m}$ below the surface on a plane inclined at $1.4 \%$ sloping towards the Léman Lake. Approximately $90 \%$ of the tunnel length is in molasse rock, which has excellent characteristics for this application, and $10 \%$ is in limestone under the Jura mountain. Internal tunnel diameter varied from $3.8 \mathrm{~m}$ in the arcs to some $5 \mathrm{~m}$ in the straight sections.

As for other accelerators considered above, the beam orbit stability was key for successful operation of the collider. Motion of few very strong superconducting focusing magnets correlated with temperature variations at the magnet support structure was found to be the main source of $\sim 3 \mathrm{~mm}$ vertical beam orbit movements [22]. Employment of local orbit correctors allowed to reduce this effect by an order of magnitude. The residual orbit motion was found to have variance growing linearly with time interval-see Fig. 10. Making the ATL law fit of Eq. (6) with coefficient $\kappa$ numerically evaluated in [23], one can estimate the diffusion constant $A_{\mathrm{LEP}}=(10.9 \pm$ 6.8) $\times 10^{-6} \mu \mathrm{m}^{2} / \mathrm{s} / \mathrm{m}[24]$.

Similar analysis has been extended for 30000 orbits which were recorded while LEP was colliding beams for its experiments in 1999 [23]. The orbit data was analyzed to reconstruct the orbit drifts that were compensated by the

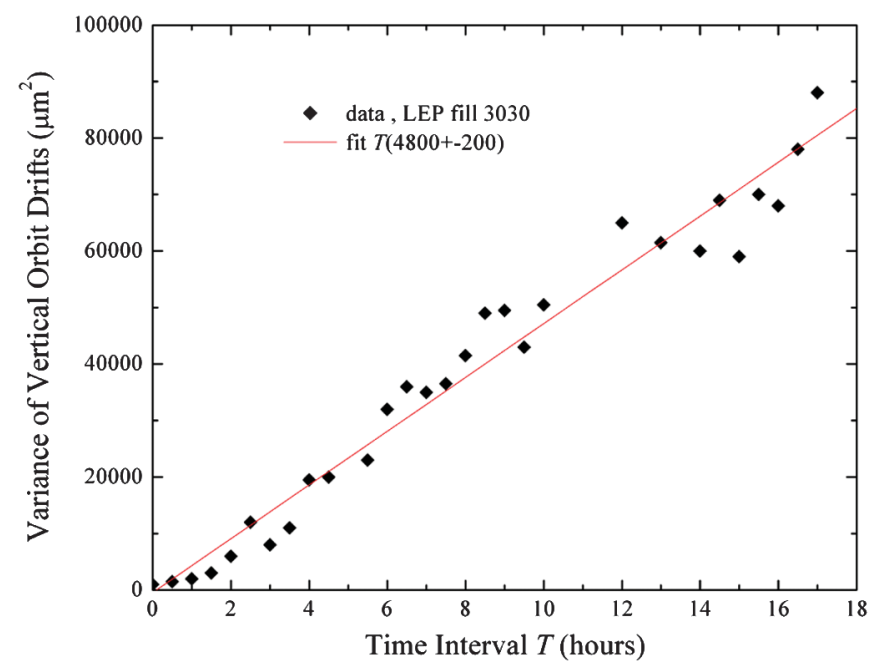

FIG. 10. (Color) Variance of the LEP vertical orbit distortions vs time interval $T$ with effects of movements of the strongest focusing magnets removed (from Refs. [22,24]).

LEP slow orbit feedback and to remove the effects due to the earth tides, motion of few very strong superconducting focusing magnets mentioned above, and other known intentional corrections implemented to optimize the accelerator operation.

Figure 11 shows the orbit root-mean square (rms) $\sigma_{V, H}$ normalized to an effective "average" BPM in the ring. The data can be very well fitted by $\sigma_{V}=(3.6 \pm$ 1.5) $[\mu \mathrm{m}] T^{1 / 2}[\mathrm{~s}]$ and $\sigma_{H}=(2.56 \pm 0.7)[\mu \mathrm{m}] T^{1 / 2}[\mathrm{~s}]$ (note significant $30 \%-40 \%$ spread in the data). Such a scaling is predicted from Eq. (6) and the diffusion coefficients can be calculated taking into account known coefficients $\kappa_{V, H}$ [23]. It is noted in Ref. [23] that, since the influence of other (unknown) effects cannot be fully excluded, then the following estimates should be considered

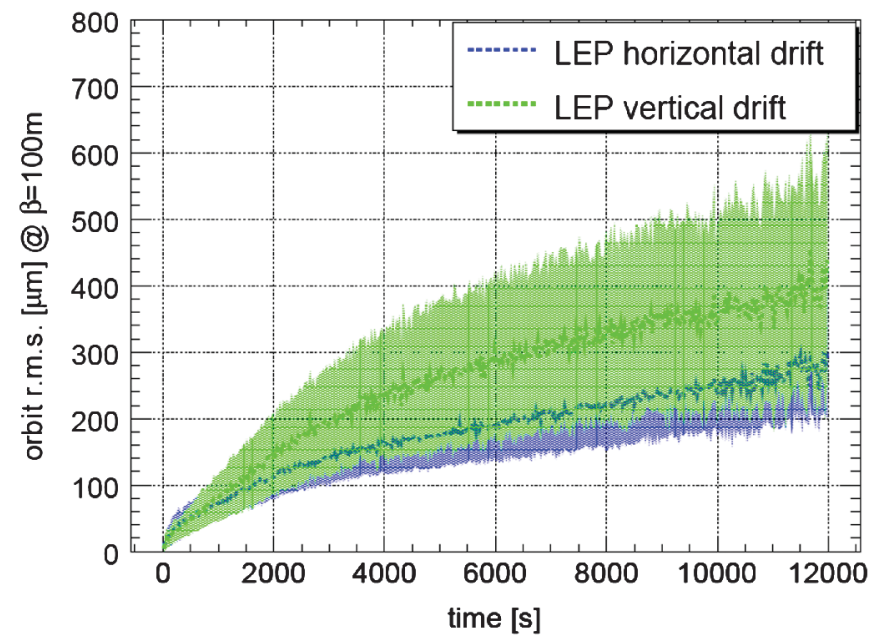

FIG. 11. (Color) The rms vertical and horizontal LEP beam orbit drifts during 1999 operation. The $\sigma \propto T^{1 / 2}$ growth with time interval $T$ is visible (from Ref. [23]). 
only as upper limits for the diffusive ground motion constants $A_{\mathrm{LEP} v}^{*}=(3.8 \pm 2.3) \times 10^{-5} \mu \mathrm{m}^{2} / \mathrm{s} / \mathrm{m} \quad$ and $A_{\mathrm{LEPh}}^{*}=(3.2 \pm 1.9) \times 10^{-5} \mu \mathrm{m}^{2} / \mathrm{s} / \mathrm{m}$.

It was reported in [25] that the tidal deformations of the Earth's crust do cause a $1 \mathrm{~mm}$ variation in the circumference of LEP. Variations of the orbit distortions over the time intervals of about 3 hours (considered in the Fig. 11 data) can be as big as $10 \%-30 \%$ of that, and were presumably taken into account in the rms orbit analysis. In addition to the periodic tidal variations, slow systematic seasonal changes of the LEP circumference of $2 \mathrm{~mm}$ have been observed. These movements might also affect the orbit analysis. They were particularly pronounced after important rainfall and might be produced by an expansion of the earth or by a pressure due to underground water levels (sponge effect) [25].

Another accelerator at CERN, named Super Proton Synchrotron (SPS), has a circumference of about $6.9 \mathrm{~km}$ and an average depth of about $50 \mathrm{~m}$. Its tunnel is embedded in the Molasse, a soft tertiary sandstone on top of a hard rock basin found in the region. The Molasse mainly consists of clay and limestone eroded from the surrounding Jura and the Alps and is covered by the Moraine, a loose and permeable more recent quaternary erosion from the Jura. In 2004, long-term SPS orbit stability measurements were performed with up to $270 \mathrm{GeV}$ proton beams. Figure 12 from Ref. [23] shows power spectra of the vertical beam motion of 270 and $26 \mathrm{GeV}$ beams that was sampled by a monitor with about $2 \mu \mathrm{m}$ rms resolution (seen as white noise above $0.1 \mathrm{~Hz}$ ).

The $26 \mathrm{GeV}$ data are thought to be dominated by slow drifts of the magnetic fields rather than by ground motion. The $270 \mathrm{GeV}$ data shows characteristic ATL-law spectrum scaling of $1 / f^{2}$. Using a precalculated vertical orbit sensitivity factor $\kappa$ for the SPS and fitting the observed orbit drifts spectra, the following SPS ground motion

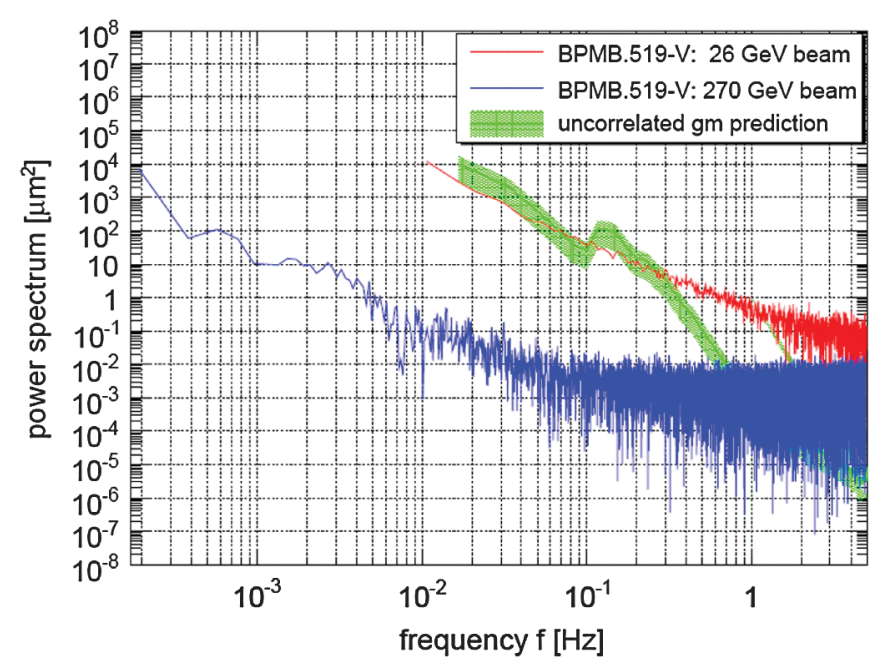

FIG. 12. (Color) Power spectra of orbit movement at 26 and $270 \mathrm{GeV}$ in the SPS (from Ref. [23]). coefficient estimate can be obtained $A_{\mathrm{SPS}}=(6.3 \pm 3) \times$ $10^{-6} \mu \mathrm{m}^{2} / \mathrm{s} / \mathrm{m}$.

\section{Ground diffusion in the accelerator alignment data}

Despite having sophisticated orbit correction systems, all accelerators undergo regular realignment of the magnets positions back their ideal values. That allows one to reduce greatly the dependence on the correction systems and helps to maintain stable operation of the facilities over periods of many years. Modern commercial instruments, e.g., laser trackers, for geodetic survey and alignment allow one to achieve accuracies of about a fraction of a $\mathrm{mm}$ over distances of a $\mathrm{km}$. A detailed description of these instruments can be found elsewhere (see, e.g., Ref. [8]). In this section we present analysis of the long-term ground motion drifts as observed during surveys and realignments of large accelerators.

\section{Long-term motion of LEP magnets}

Several times a year, positions of more than 700 focusing magnets of the LEP were measured and restored back to their prescribed values to follow an ideal smooth curve. Results of four measurements of the LEP magnet elevations in 1993-1994 [26] are shown in Fig. 13.

The average tilt of $1.4 \%$ was subtracted from the data. For the purpose of the presentation in one figure, the four curves are vertically separated by $2 \mathrm{~mm}$ from each other. The top line in Fig. 13 shows vertical positions the magnets in April 30, 1993, just after making the realignment of the accelerator to a smooth curve. The roughness of this curve is thought to be mostly due to the instrumentation accuracy. Some 9 months after the April 1993 realignment, on

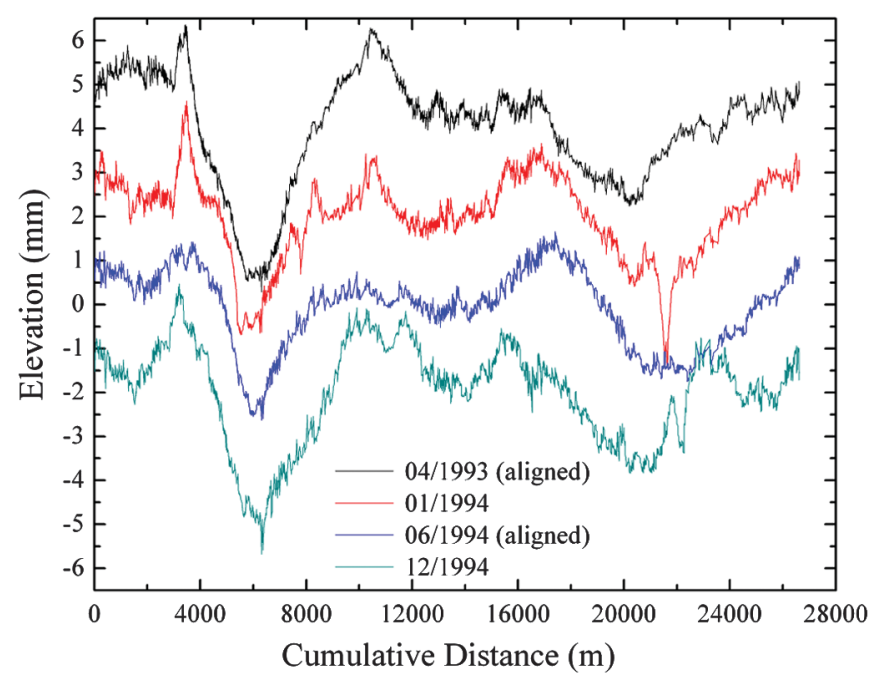

FIG. 13. (Color) Elevations of the CERN's LEP focusing magnets measured in 1993-1994 [26] vs cumulative distance along the ring (i.e., the point at $0 \mathrm{~m}$ placed close to the point at $26.7 \mathrm{~km}$, data courtesy of Jean-Pierre Quesnel of the CERN's Survey Group). 
January 28, 1994, the positions had been remeasured-see the 2 nd from the top line. One can see that the line is more rough and several peaks have appeared, the biggest are around 3500 and $21500 \mathrm{~m}$ which are the regions of systematic long-term drifts due to well-known geological instability. Then, the realignment had been done and the LEP magnets elevations as measured June 6, 1994 are presented in the 3rd line from the top. Major peaks are now smoothed. Six months after, in December 1994, they reappear, see the bottom line in Fig. 13, together with other smaller changes. Further analysis and data processing reported in Ref. [17], include: (1) $1 \mathrm{~km}$ long pieces of the LEP circumference around 3500 and $21500 \mathrm{~m}$ were ex- cluded from the analysis (as they had been dominated by systematic drifts); (2) the lowest five Fourier harmonics were subtracted from all the data sets (as they did not represent any ground motion at all-they are large amplitude smooth spatial curves which the surveyors used as the targeted lines to get all the magnets on for efficient collider operation). Then, the variances of the first difference $\left\langle d Y^{2}(L)\right\rangle=\left\langle[d Y(l)-d Y(l+L)]^{2}\right\rangle$ have been calculated, and, as usual, the brackets $\langle\cdots\rangle$ denote averaging over all possible pairs of the magnets distanced by $L$. The results are presented in Fig. 14. The straight lines represent linear fits:

$$
\begin{array}{rlrl}
\text { April 30, 1993 } & \left\langle d Y^{2}(L)\right\rangle_{\mathrm{I}} & =(0.0147 \pm 0.0014)+L\left(1.63 \times 10^{-4} \pm 2.4 \times 10^{-6}\right), \\
\text { January 28, 1994 } & \left\langle d Y^{2}(L)\right\rangle_{\mathrm{II}} & =(0.0218 \pm 0.005)+L\left(3.72 \times 10^{-4} \pm 8.9 \times 10^{-6}\right), \\
\text { June 6, 1994 } & \left\langle d Y^{2}(L)\right\rangle_{\mathrm{III}}=(0.0001 \pm 0.0043)+L\left(2.36 \times 10^{-4} \pm 7.3 \times 10^{-6}\right), \\
\text { December 1994 } & \left\langle d Y^{2}(L)\right\rangle_{\mathrm{IV}}=(0.017 \pm 0.005)+L\left(3.42 \times 10^{-4} \pm 9.2 \times 10^{-6}\right) .
\end{array}
$$

One can see that, for $L<1000 \mathrm{~m}$, the variances for the just-recently realigned accelerator $\left\langle d Y^{2}(L)\right\rangle_{\mathrm{I}}$ and $\left\langle d Y^{2}(L)\right\rangle_{\mathrm{III}}$ are 1.5-2 times less than what is measured after several months without alignment $\left\langle d Y^{2}(L)\right\rangle_{\mathrm{II}}$ and $\left\langle d Y^{2}(L)\right\rangle_{\mathrm{IV}}$. It has to be noticed that the variance grows linearly with $L$ even in the cases of recently aligned magnets $\left\langle d Y^{2}(L)\right\rangle_{\mathrm{I}}$ and $\left\langle d Y^{2}(L)\right\rangle_{\mathrm{III}}$. That is because of the sequential method of the survey-one segment of the machine was measured and used as the reference for another. Therefore, the random errors of the position measurement of a given magnet with respect to the previous one add up like a random walk. Such a random walk error can be estimated by the closure errors of about $2 \mathrm{~mm}$ over

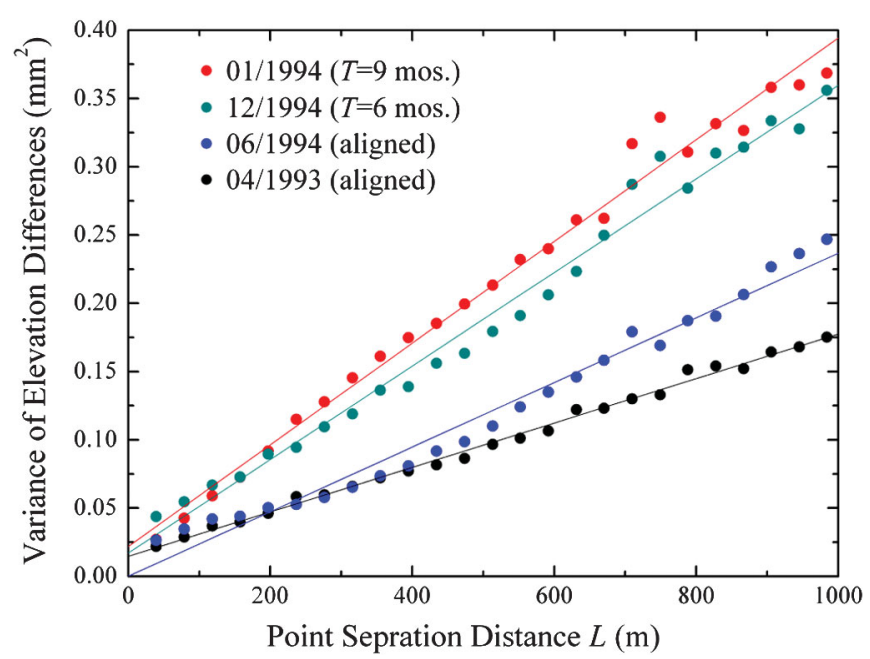

FIG. 14. (Color) The variance of relative displacement of the CERN LEP magnets vs the distance between them $L$ (from Ref. [17]). the entire circumference (measured at different periods) that is equivalent to $0.14 \mathrm{~mm}^{2} / \mathrm{km}$-in good agreement with the analysis shown in Fig. 14. The increase of the variance after the time interval (the top two lines) over the instrumentation noise (the bottom two lines) is a clear indication of the ground diffusion which took place in between the measurements. Again, assuming validity of the ATL law, one gets two estimates of the diffusion constant $A$ :

$$
\begin{aligned}
A_{\mathrm{II}-\mathrm{I}} & =\frac{\left\langle d Y^{2}(L)\right\rangle_{\mathrm{II}}-\left\langle d Y^{2}(L)\right\rangle_{\mathrm{I}}}{L \cdot 9 \text { months }} \\
& =(9.0 \pm 0.5) \times 10^{-6} \frac{\mu \mathrm{m}^{2}}{\mathrm{~s} \cdot \mathrm{m}}, \\
A_{\mathrm{IV}-\mathrm{III}} & =\frac{\left\langle d Y^{2}(L)\right\rangle_{\mathrm{IV}}-\left\langle d Y^{2}(L)\right\rangle_{\mathrm{III}}}{L \cdot 6 \text { months }} \\
& =(6.8 \pm 0.8) \times 10^{-6} \frac{\mu \mathrm{m}^{2}}{\mathrm{~s} \mathrm{~m}},
\end{aligned}
$$

which are remarkably close to each other. Therefore, the LEP alignment data demonstrate that the variance of the relative displacements in time scales proportionally to the distance between the points. Much longer, six-year elevation changes of the LEP magnets in 1993-1999 have been analyzed in Ref. [27]. It was shown that, after exclusion of the linear trends and systematic drifts from the data, the remaining random diffusion can be described by the ATL law with coefficient $A_{\mathrm{LEP}}=(2.9 \pm 0.6) \times$ $10^{-6} \mu \mathrm{m}^{2} / \mathrm{s} / \mathrm{m}$.

\section{Motion of CERN's Super Proton Synchrotron magnets}

The noted above CERN's Super Proton Synchrotron (SPS) was constructed in the mid-1970s and has a $6.9 \mathrm{~km}$ 


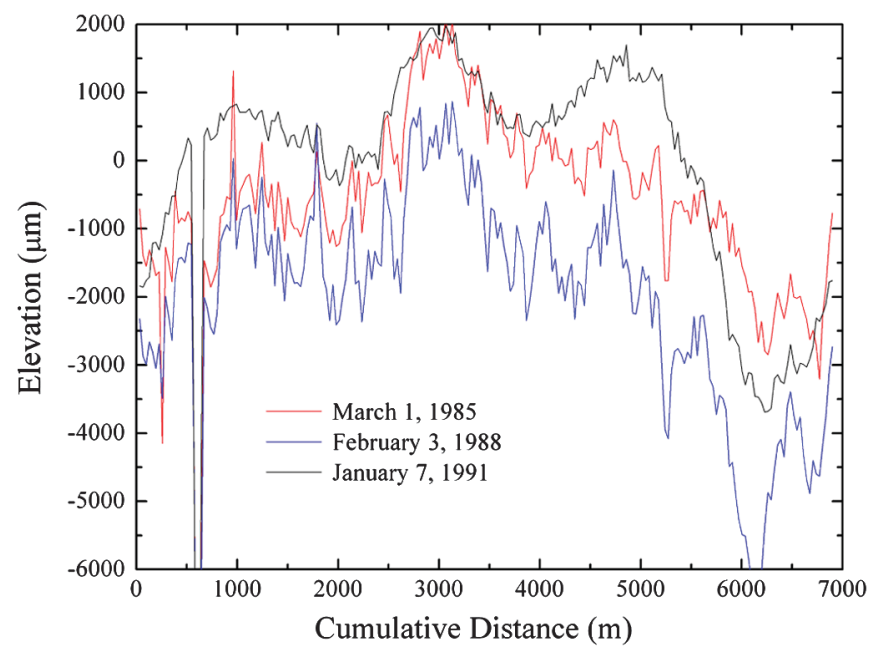

FIG. 15. (Color) Displacements of the CERN's Super Proton Synchrotron magnets measured in 1985, 1988, and 1991 along the circumference ring; the point at $0 \mathrm{~m}$ placed close to the point at 6912 m (data courtesy of Jean-Pierre Quesnel of the CERN's Survey Group).

circumference. There are 744 bending magnets and $N=$ 216 focusing magnets placed practically uniformly over the ring. Primary data from an optical survey shown in Fig. 15 represent the vertical displacements of the magnets relative to the theoretical ideal position of 1976. These values were measured 3 times at about three-year intervals: in 1985, 1988, and 1991—with estimated accuracy of about few dozens of micrometers.

The SPS data were processed in a way similar to the one for the LEP alignment data discussed above, so, for example, the data for several magnets around $600 \mathrm{~m}$ and fewer were not taken into consideration as these magnets were intentionally displaced during the period.

The variances of the relative vertical displacements of the magnets versus distance $L$ are presented in Fig. 16 from [28] together with linear fits (dashed lines) according to the ATL law with diffusion coefficients of $20 \times$ $10^{-6} \mu \mathrm{m}^{2} / \mathrm{s} / \mathrm{m}, \quad 40 \times 10^{-6} \mu \mathrm{m}^{2} / \mathrm{s} / \mathrm{m}, \quad 10 \times$ $10^{-6} \mu \mathrm{m}^{2} / \mathrm{s} / \mathrm{m}$, and $13 \times 10^{-6} \mu \mathrm{m}^{2} / \mathrm{s} / \mathrm{m}$ for time intervals of 1985-1988, 1988-1991, 1985-1991, and 19761988, correspondingly. It has to be emphasized that the time intervals vary from 3 to 12 years, and nevertheless the diffusive constants are almost the same. An average value of the coefficient for the SPS data is $A_{\mathrm{SPS}}=(14 \pm 5) \times$ $10^{-6} \mu \mathrm{m}^{2} / \mathrm{s} / \mathrm{m}$. Note that a power-law fit $\left\langle d Y^{2}(L)\right\rangle \propto L^{\gamma}$ with exponent $\gamma$ less than 1 might better describe the variance shown in Fig. 16(b) than the linear fit.

\section{Tevatron alignment data analysis}

Alignment system of the Tevatron Collider employs more than 200 geodetic "tie rods" installed in the concrete tunnel wall all over the ring, approximately $30 \mathrm{~m}$ apart.
Position of the magnets is regularly locally referenced with respect to the rods while positions of the rods are routinely globally monitored. The rod elevations data are available for the years of 2001, 2003, 2005, 2006, and 2007. Figure 17 shows the change of the elevations around the ring accumulated over two intervals- two years (20032005) and six years (2001-2007). One can see that the longer term motion has larger amplitude. The variance $\left\langle d Y^{2}(L)\right\rangle=\left\langle[d Y(z)-d Y(z+L)]^{2}\right\rangle$ of the displacements has been calculated and averaged over all possible time intervals. For example, there are two one-year intervals (2005-2006, 2006-2007), three two-year intervals (20012003, 2003-2005, 2005-2007), etc., and one for the sixyear interval 2001-2007. The results for one-year changes and for the six-year change are shown in Fig. 18. A remarkable difference between the two plots is that $1 \mathrm{yr}$ variance scales linearly only up to $L \approx 900 \mathrm{~m}$ and does not depend on $L$ beyond that scale, while the $6 \mathrm{yr}$ variance grows all the way to distances as large as $1800 \mathrm{~m}[29,30]$. Such a behavior indicates independence of the displacements of the rods located more than $900 \mathrm{~m}$ apart on the time scale of a year, and existence of a significant level of interdependence of the motion of distanced rods at the times as long as six years. The calculated variances for all possible time difference can be well approximated by linear fits $\left\langle d Y^{2}(L)\right\rangle=a+b L$ over distances less than $900 \mathrm{~m}$ and the slopes (fit parameters $b$ with the error bars) are plotted in Fig. 19.

One can see that the variance per unit of the distance grows with the time interval between the measurements, and can be approximated by a linear fit $b(T)=c T$ with $c=0.153 \pm 0.004\left[\mathrm{~mm}^{2} / \mathrm{km} /\right.$ year $]$. Such dependence is in accordance with the ATL law with coefficient $A_{\text {Tevatron }}=c=(4.9 \pm 0.13) \times 10^{-6} \mu \mathrm{m}^{2} / \mathrm{s} / \mathrm{m}[30]$.

\section{Alignment data on ground motion in other accelerators}

The variance of the 1985-1988 SPS elevation changes is compared with the alignment data from several other accelerator sites in Fig. 20 from Ref. [28]. Because of the different times of observations for these data, they are presented as functions of the variance of displacement divided by the time of observations vs distance $L$ between the points of the ground. For comparison, the ATL-law scaling with coefficient $A=100 \times 10^{-6} \mu \mathrm{m}^{2} / \mathrm{s} / \mathrm{m}$ is also shown by a dashed line. That line well approximates the theodolite measurements of vertical movements of few dozen surface monuments along a $2 \mathrm{~km}$ long straight line at the UNK collider construction site (Protvino, Moscow region, Russia) made over time interval $T$ of about two years.

The other two lines represent the data of the measurements made at the Stanford Linear Accelerator Center (SLAC) accelerators: one for the $2 \mathrm{~km}$ circumference PEP accelerator magnet displacements during 20 months (1989-1991) and another is for very long-term displace- 
(a)

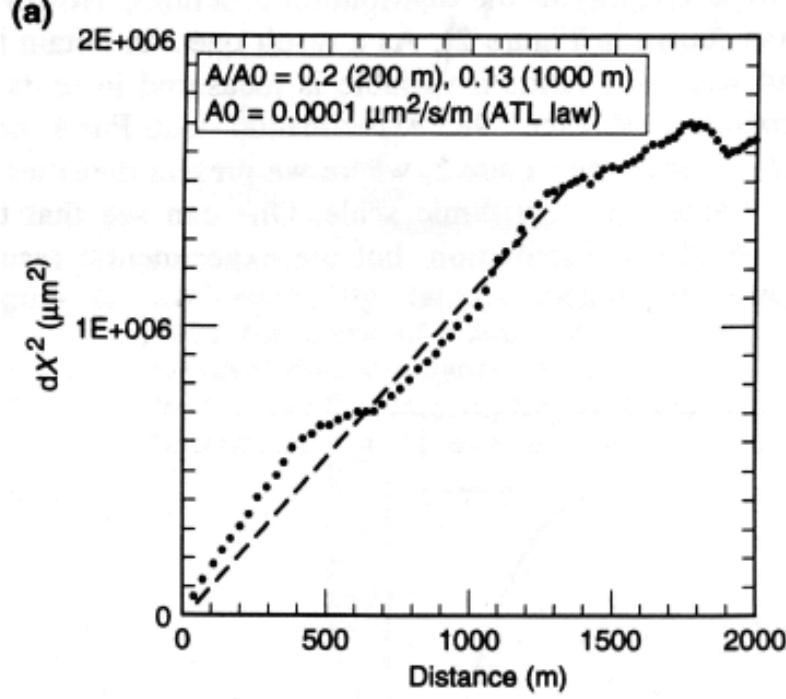

(b)

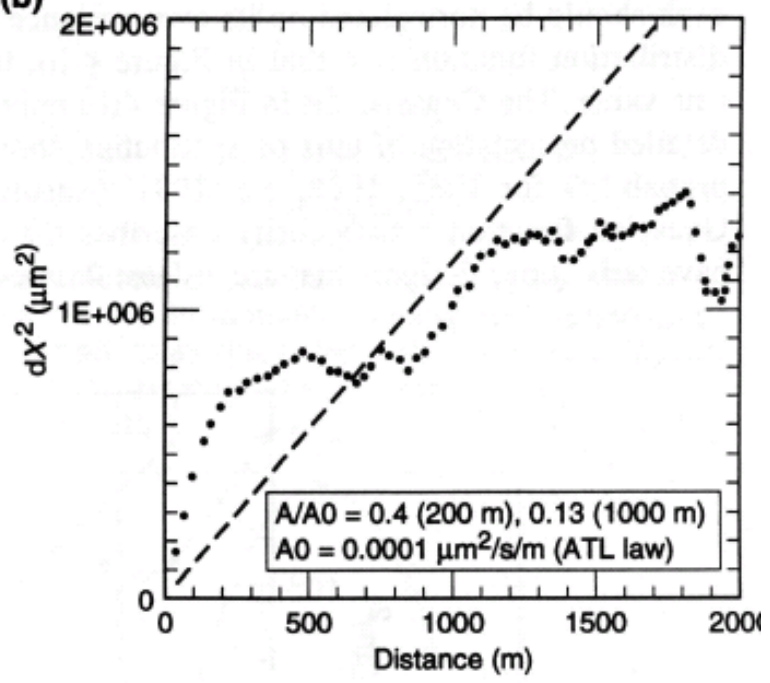

(c)

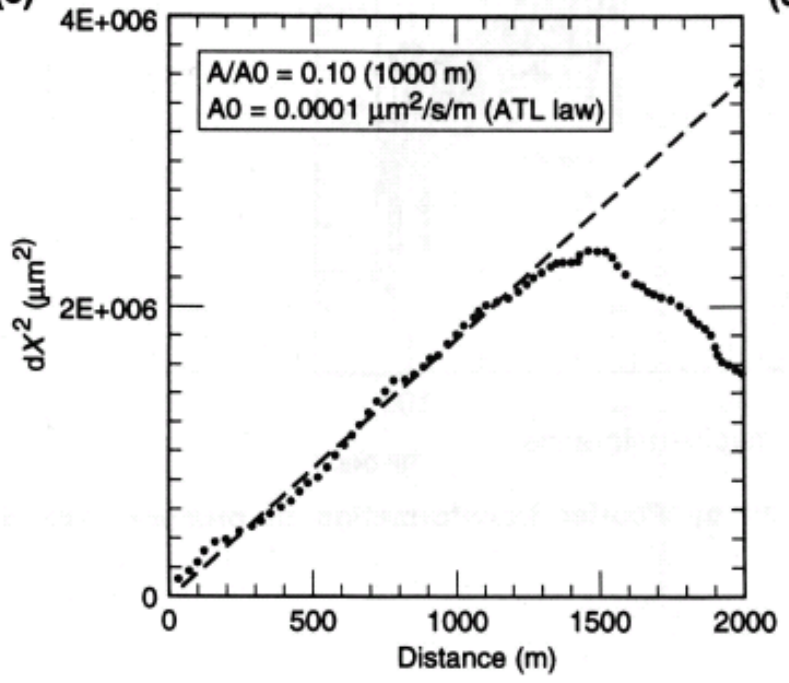

(d)

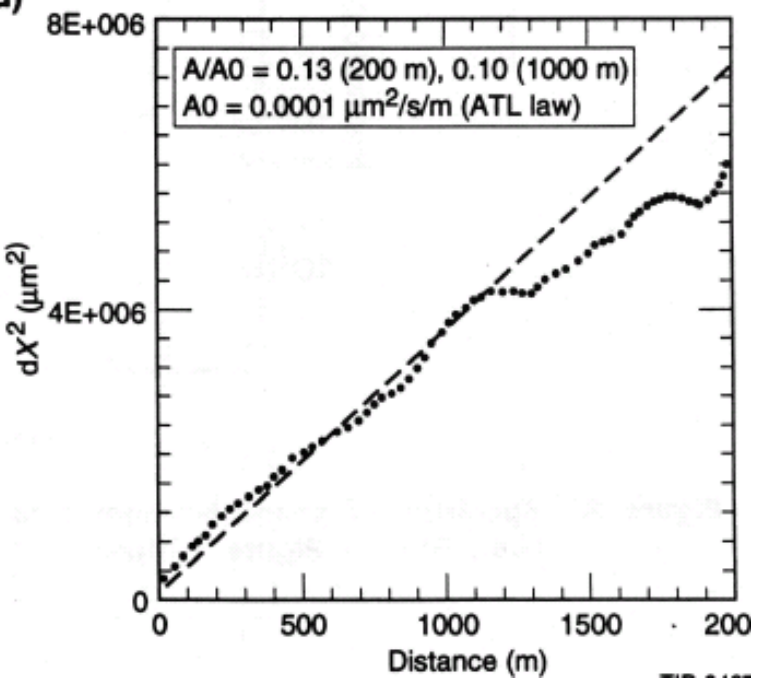

FIG. 16. The variance of the relative vertical displacement of the SPS magnets after various time intervals vs distance between the points of the position survey L: (a) three years (1985-1988), (b) three years (1988-1991), (c) six years (1985-1991), (d) 12 years (1976-1988) (from Ref. [28]).

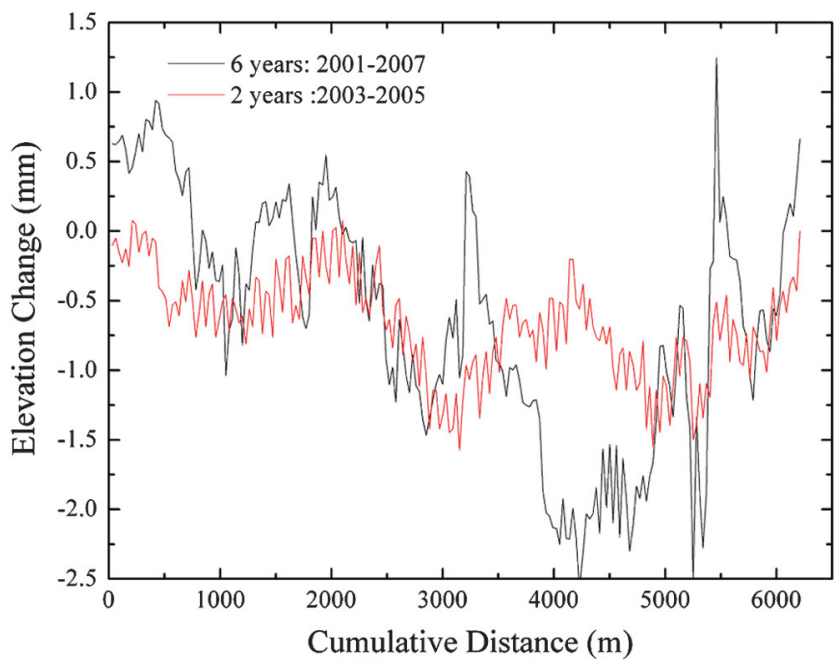

FIG. 17. (Color) Vertical displacement of more than 200 "tie rods" in the Tevatron tunnel over the period of 2003-2005 and a 6 yr period of 2001-2007 (data courtesy of James Volk and Fermilab's Alignment Group). 


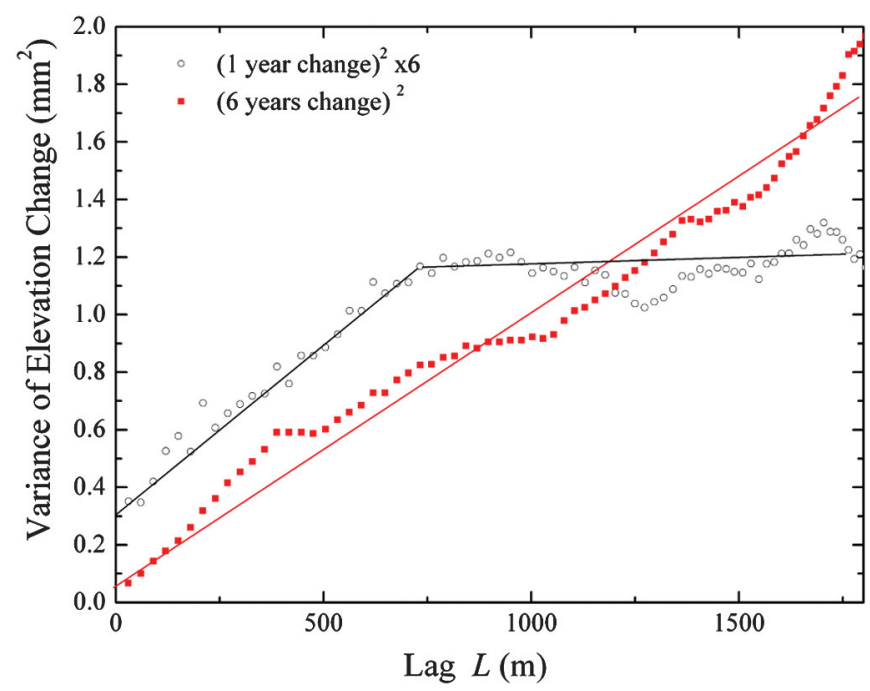

FIG. 18. (Color) Variances of the averaged Tevatron tie rod vertical displacements over time intervals of one (multiplied by 6) and six years vs the distance $L$ (from Ref. [29,30]).

ments over 17 years of the elements in a two-mile linear accelerator tunnel. These tunnels in SLAC sit on or are mined in grey unweathered well cemented tertiary myocene sandstone. Possibly due to a "cut and cover" construction method and smaller depth, the SLAC linac tunnel demonstrates faster diffusion than the PEP tunnel-the coefficients are $A_{\mathrm{SLC}}=(200 \pm 100) \times 10^{-6} \mu \mathrm{m}^{2} / \mathrm{s} / \mathrm{m}$ and $A_{\mathrm{PEP}}=(100 \pm 50) \times 10^{-6} \mu \mathrm{m}^{2} / \mathrm{s} / \mathrm{m}$ correspondingly. Much lower diffusion in the SPS tunnel can be explained by the comparatively low depth of the SPS and the relatively hard rock at the CERN cite. It has also been recently pointed out that if a long-term systematic motion is excluded then a purely diffusive component of the SLAC

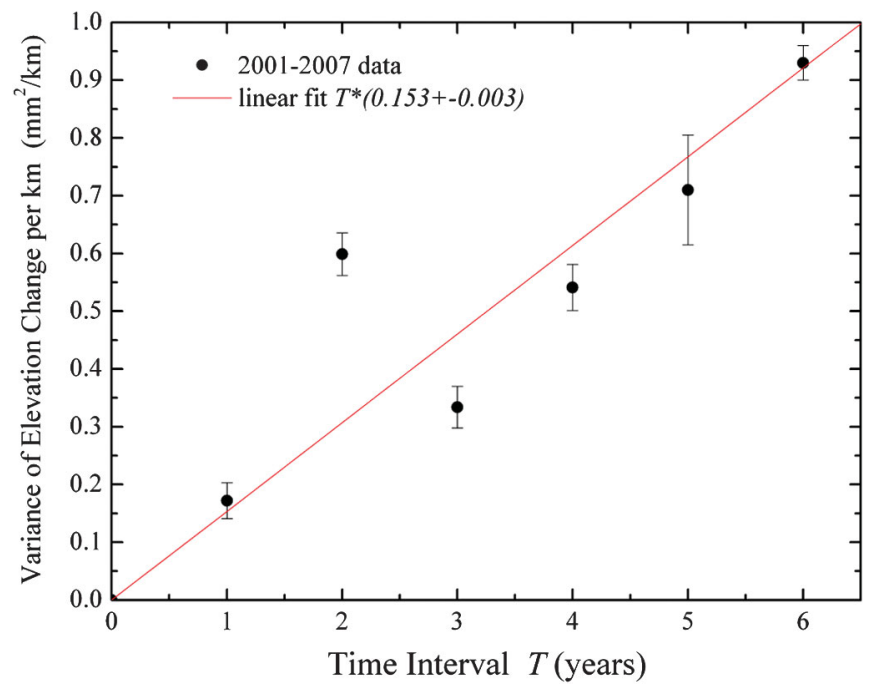

FIG. 19. (Color) Variances of the Tevatron alignment rod displacements per unit distance vs the time interval between the measurements (see text, from Ref. [29,30]).

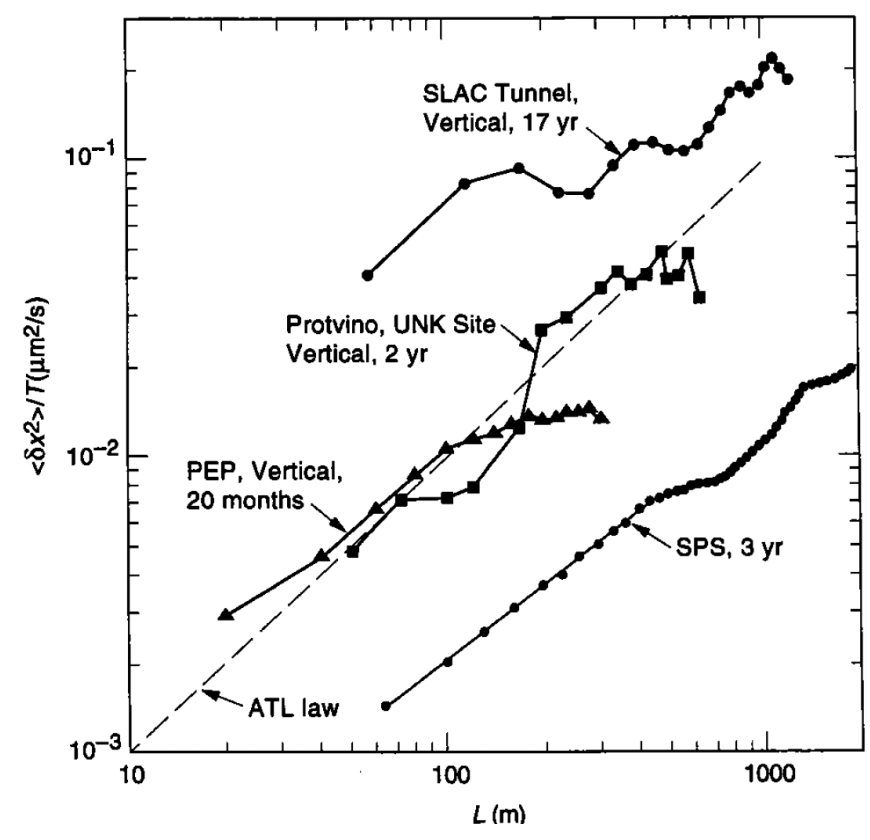

FIG. 20. Variances of the accelerator magnet displacements per unit time vs distance for the SLC, UNK, PEP, and SLAC tunnel (see text, from Ref. [28]).

linear accelerator tunnel motion exhibits a much lower diffusion coefficient $A_{\mathrm{SLC}}<10 \times 10^{-6} \mu \mathrm{m}^{2} / \mathrm{s} / \mathrm{m}$ [31]. It has to be noted also that for all the data presented in Fig. 20 the exponent $\gamma$ of a power-law fit $\left\langle d Y^{2}(L)\right\rangle \propto L^{\gamma}$ varies between 0.7 and 1.0.

\section{Geophysics measurements data on ground diffusion}

Evidence of the ground diffusion either in space or in time or simultaneously in space and time have been reported in geophysics studies of various types. Below we present many of these results, classifying them by the method of the measurements: made with optical and laser interferometers, stretched wire, and several types of HLSs.

\section{Strain measurements in PFO}

Horizontal motion of massive near surface monuments emplaced in competent, weathered granite has been made by laser interferometers ("optical anchors") at Pinon Flat Observatory (PFO) in southern California [9]. The data on the optical path difference $d L$ over the distance $L=732 \mathrm{~m}$ have been normalized in the units of strain $\varepsilon=(d L / L)$ and its power spectral density is shown in Fig. 21 from [32]. The peaks in the spectrum around multiples of 1 cycle/day are caused by earth tides and temperature effects; the peak at high frequencies of $\sim 0.1 \mathrm{~Hz}$ is caused by microseisms (the "7-second hum"). Except for these peaks, the spectrum is very well fit by the power law $1 / f^{2}$. Correspondingly, the mean square of the strain variations $\left\langle[\varepsilon(t)-\varepsilon(t+T)]^{2}\right\rangle$ scales linearly with time $T$ as demonstrated in the lower plot of Fig. 21. From the linear slope, 

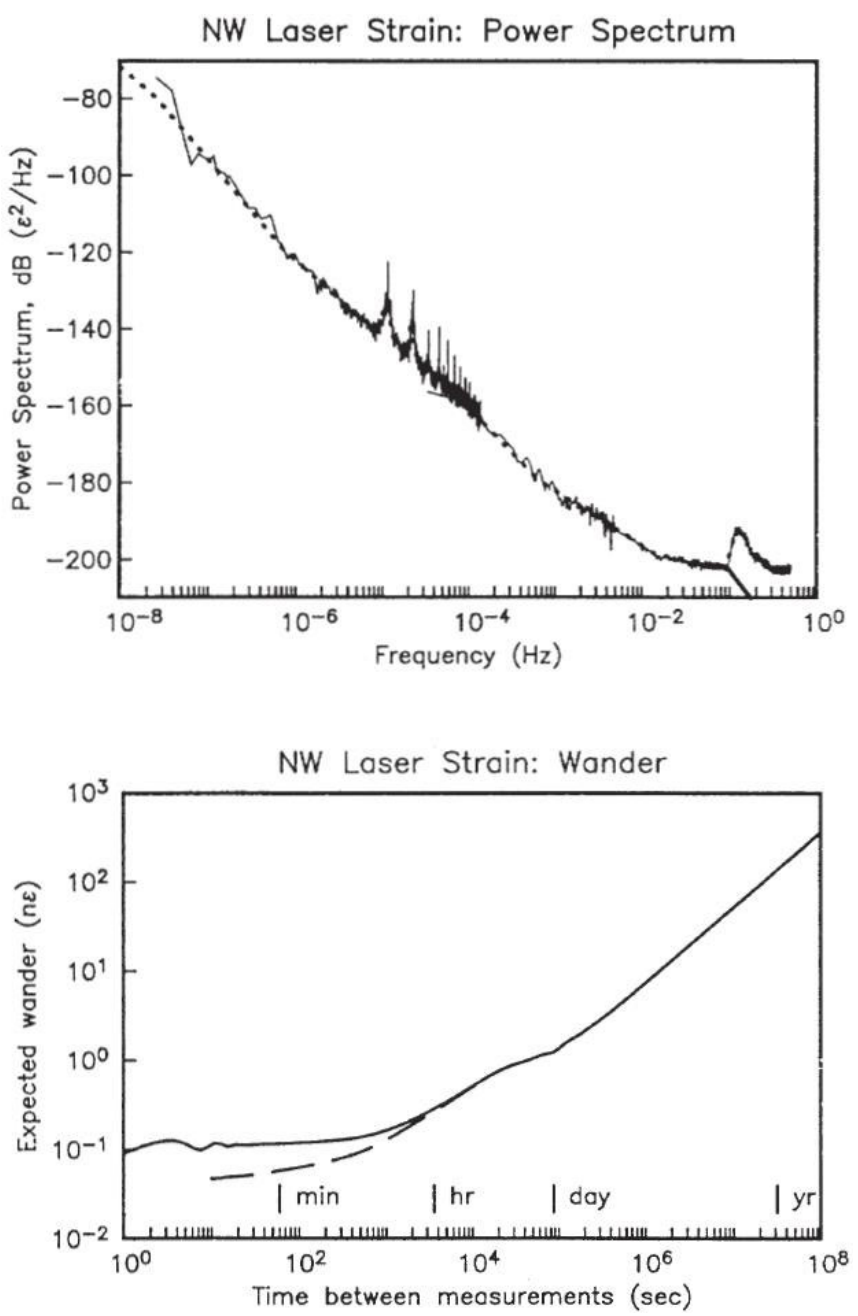

FIG. 21. (top) PSD of the earth strain at Pino Flat Observatory in southern California; (bottom) the solid line is rms wander of the earth computed from the full spectrum, and that computed if the "7-second" microseism peak is filtered out, from [32].

the ATL coefficient can be calculated as $A_{\mathrm{PFO}}=\langle[\varepsilon(t)-$ $\left.\varepsilon(t+T)]^{2}\right\rangle \times L / T=0.7 \times 10^{-6} \mu \mathrm{m}^{2} / \mathrm{s} / \mathrm{m}$. This diffusion coefficient is very small compared to any examples we considered above - that is no surprise given that the PFO has been located in a very stable area with hard granite bedrock suitable for very precise geophysics observations.

\section{Laser alignment system in the SLAC tunnel}

Several measurements of slow ground motion were performed using the laser alignment system [33] installed in the SLAC two-mile linear accelerator tunnel. This system consists of a light source, a detector, and about 300 targets, one of which is located at each point to be aligned over a total length of $3050 \mathrm{~m}$. The target is a rectangular Fresnel lens which has pneumatic actuators that allow each lens to be flipped in or out. The targets are installed in a two-foot

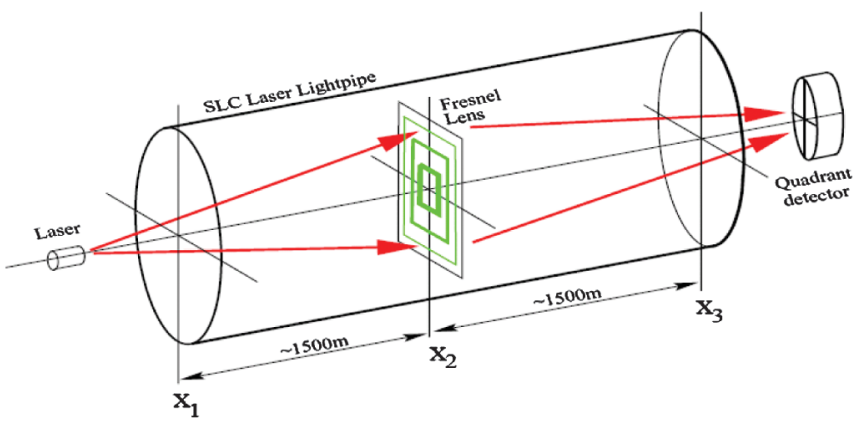

FIG. 22. (Color) Schematic of SLAC linear accelerator laser measurement system.

diameter aluminum pipe which is the basic support girder for the SLAC linear accelerator.

The light source is a He-Ne laser shining through a pinhole diaphragm. The beam divergence is large enough to cover even nearby targets and only transverse position of the laser, but not angle, influences the image position. The light pipe is evacuated to about 15 microns of $\mathrm{Hg}$ to prevent deflection of the alignment image due to refraction in air. Sections of the light pipe, which are about 12 meters long, are connected via bellows that allow independent motion or adjustment. The measurements reported in $[34,35]$ were done with a single lens inserted which was not moved until the measurements were finished in order to ensure maximal accuracy. (In multitarget mode the repeatability of the target positioning limits the accuracy.) The schematic of the measurements with just one of the lenses exactly in the middle of the system is shown in Fig. 22. In such a configuration, the laser spot position in the detector is equal to $x_{1}+x_{3}-2 x_{2}$ (for either vertical or horizontal plane-see Fig. 22).

Analysis of the spot's vertical position variation shows that the variance of the motion scales linearly with time-

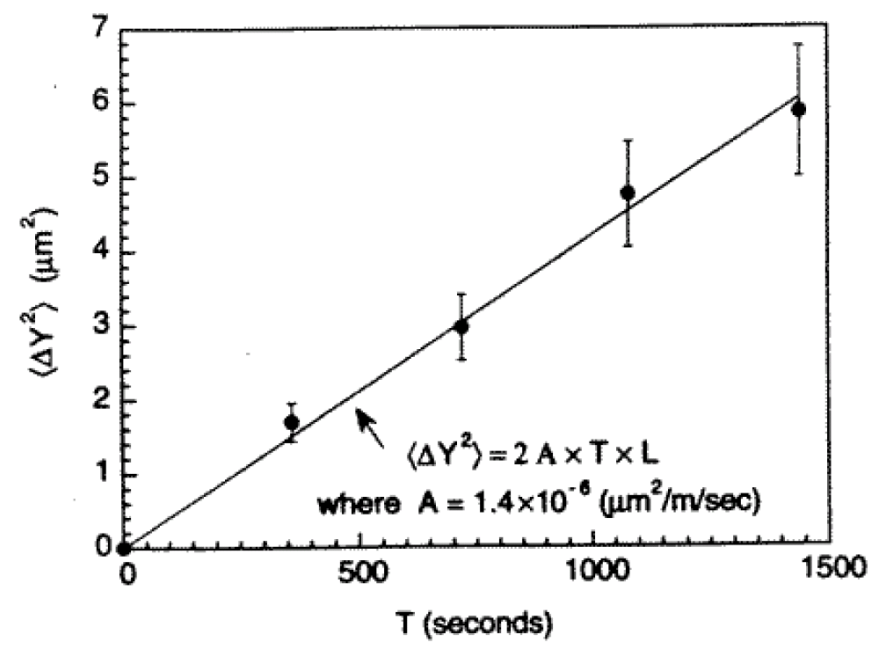

FIG. 23. Variance of the vertical laser spot movement in the SLAC laser system (from Ref. [34]). 


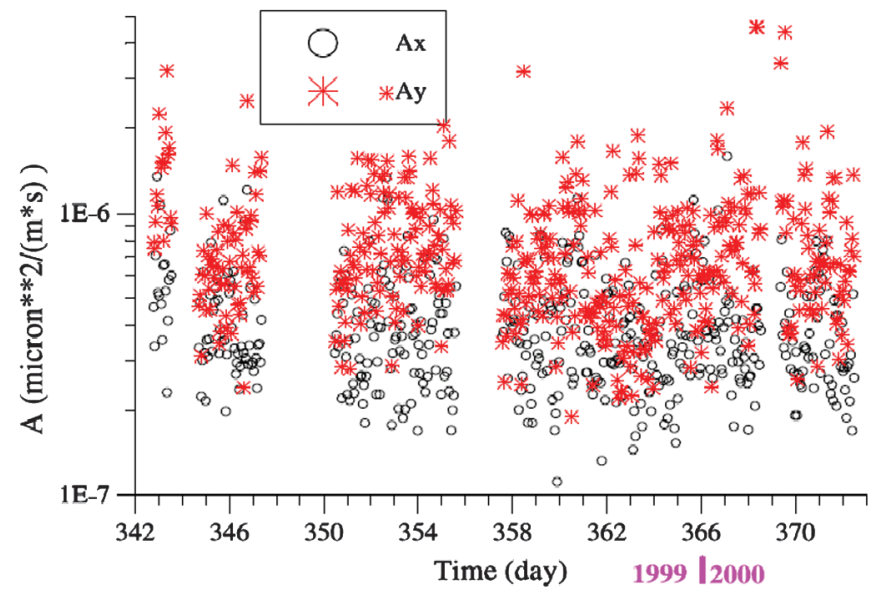

FIG. 24. (Color) Diffusion coefficient $A$ as measured from the spectra laser spot vertical and horizontal movements in the frequency band 0.00024 to $0.015 \mathrm{~Hz}$ (from Ref. [35]).

see Fig. 23 from Ref. [34]-that is consistent with $A_{\text {SLAC }}=1.4 \times 10^{-6} \mu \mathrm{m}^{2} / \mathrm{s} / \mathrm{m}$.

In a separate series of measurements [35], it was found that the amplitudes of the diffusive motion in the vertical and horizontal planes are about the same, see Fig. 24, and the excess in the vertical plane is often correlated with the atmospheric pressure variations.

\section{Motion of the CERN PS pillar}

Yet another manifestation of the ground diffusion is the movement of central CERN proton synchrotron (PS) pillar over a period of more than two years, shown in Fig. 25 from Ref. [36].

A pair of horizontal pendulums was mounted on the PS pillar anchored in the molasse $10 \mathrm{~m}$ below ground level. These instruments measure the variations of their support in relation to the direction of the vertical, and, therefore, the movement of the vertical axis of the $10 \mathrm{~m}$ deep pillar. Such an inverted pendulum performed irregular motion that looks like Brownian motion. After subtracting a linear trend (well seen in the South-North direction), one can find that in both directions the variance grows about linearly in time, and the coefficients of the ATL diffusion are equal to $A_{\mathrm{PS}}=(3.0 \pm 1.0) \times 10^{-6} \mu \mathrm{m}^{2} / \mathrm{s} / \mathrm{m}$, which corresponds to the variance of displacement of about $500-900 \mu \mathrm{m}^{2}$ over the time interval of $T=9$ months and $L=10 \mathrm{~m}$ [37].

\section{Stretched wire measurements at the SLAC FFTB facility}

Stretched wires were used for measurements of vertical and horizontal positions of several magnets in SLAC final focus test beam (FFTB) tunnel [38]. The magnets were divided into four sections with two parallel stretched wires in each section ("left" and "right" wires). The wire lengths vary from $30 \mathrm{~m}$ to about $43 \mathrm{~m}$ in the different

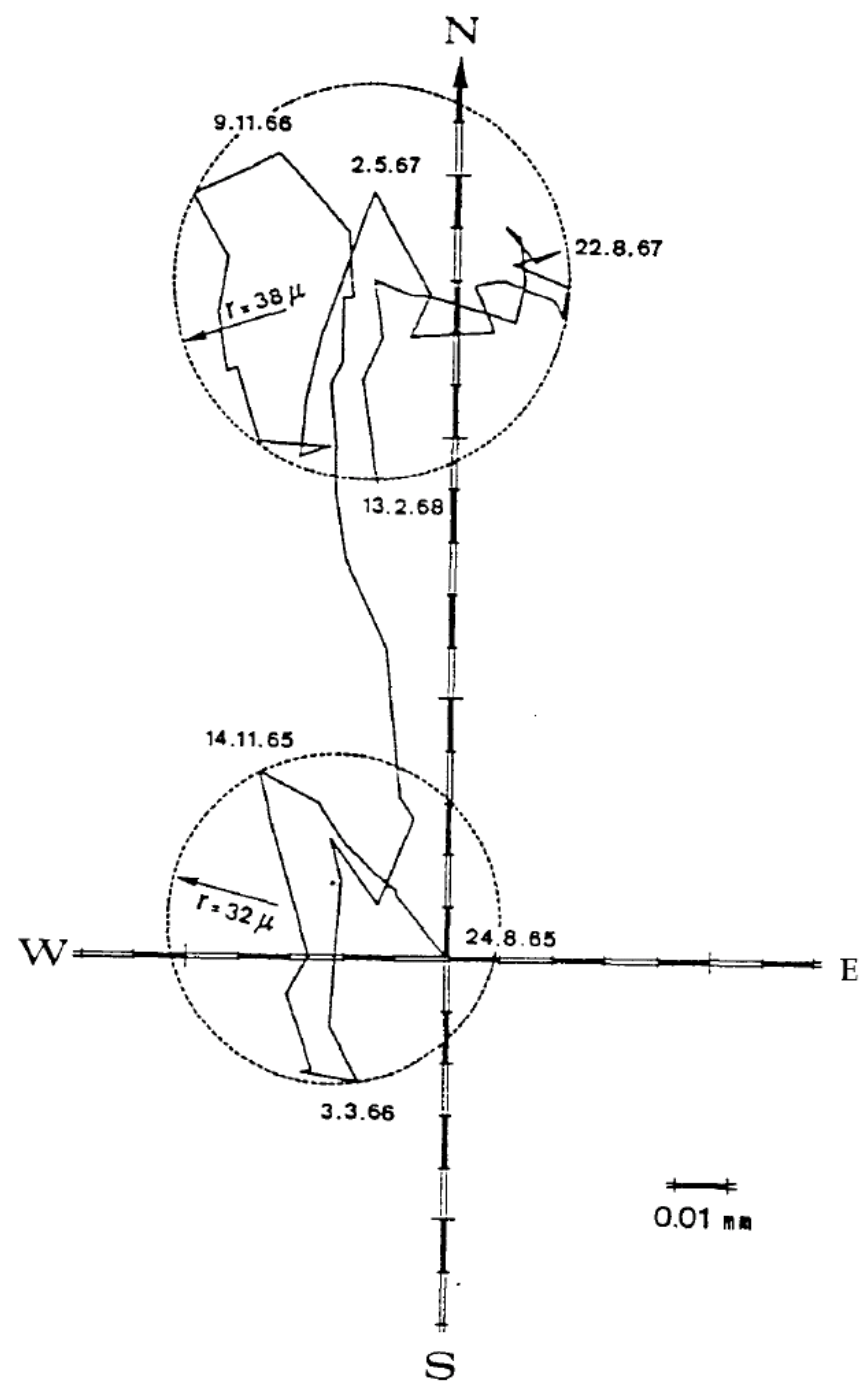

FIG. 25. Horizontal movement of the PS central pillar in 1965-1968 (from Ref. [36]).

wire sections. Each wire was stretched with a weight of about $35 \mathrm{~kg}$ at one end. Each magnet had submicron resolution wire position monitors attached to it. The measurements were taken over about a week in the FFTB hall with a measurement point every 6 seconds. The hall has a thick concrete slab floor and was sealed to avoid thermal variations for most of the measurement interval. The results shown in Fig. 26 indicate that the element positions wander in both vertical and horizontal planes with the diffusion coefficients in the range $A_{\mathrm{FFTB}}=(4 \pm 3) \times$ $10^{-6} \mu \mathrm{m}^{2} / \mathrm{s} / \mathrm{m}$.

\section{HLS measurements in Japan}

Below we review several slow ground motion measurements made with HLS sensors made in various locations in Japan: in geophysics laboratories, in accelerator facilities, and in several tunnels. More detail descriptions of the 


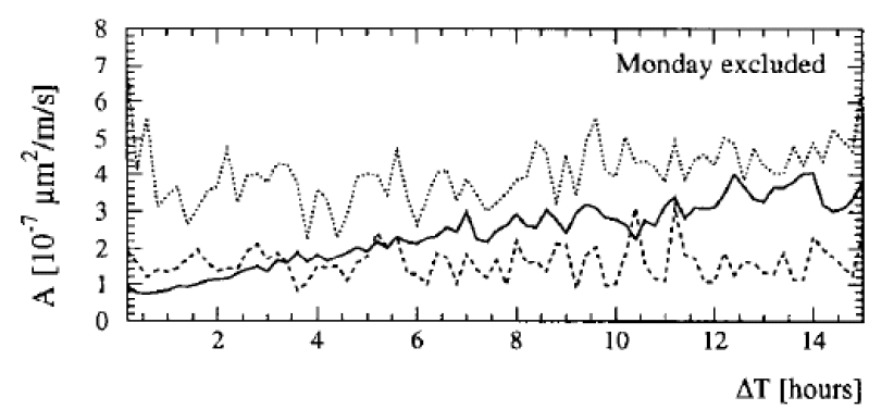

FIG. 26. Calculated diffusion constant $A$ as a function of the time interval $\Delta T$ in the ATL rule. The three different curves refer to the horizontal (solid) and vertical (dotted) data of Sec. I and the horizontal data of Sec. II (dashed line). The $A$ constant was determined over a distance of about twice $15 \mathrm{~m}$ (from Ref. [38]).

conditions and instruments can be found in the cited references.

\section{a. Esashi Earth tide station}

The Esashi Earth tide station is situated in the northwest of Japan. It occupies a tunnel in a granite mountain side. Two $L=50-\mathrm{m}$ long water levels directed to South-North and East-West are at about $160 \mathrm{~m}$ from the tunnel entrance and about $60 \mathrm{~m}$ under the mountain surface. These tiltmeters detect vertical elevation difference. Observations started in June 1979 by National Astronomical Observatory Mizusawa. Figure 27 presents an almost 15years-long record of S-N and E-W tilts measured monthly [39]. Linear trends were extracted from the original data records and the variogram of the tilt $\left\langle d \Theta^{2}(T)\right\rangle=\langle[\Theta(t)-$ $\left.\Theta(t+T)]^{2}\right\rangle$ calculated in [17]. The results are presented in Fig. 28 and the data can be approximated by the linear fits of $0.026 \mu \mathrm{rad}^{2} /$ month for the N-S tilt data and

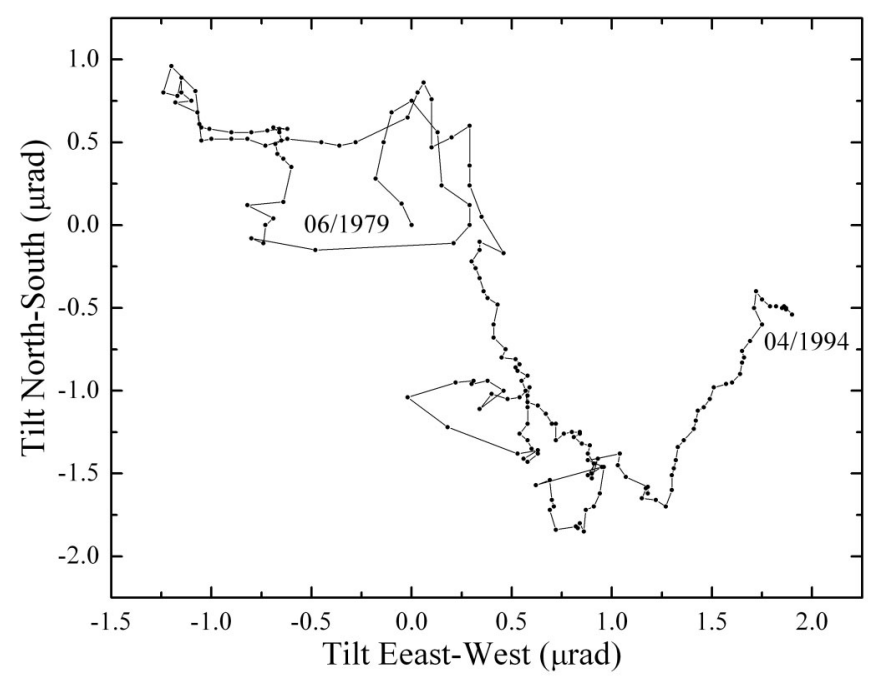

FIG. 27. Secular tilting motion measured at the Esashi station in 1979-1994 (from Ref [39], original data records courtesy of Professor Shigeryu Takeda of KEK, Japan).

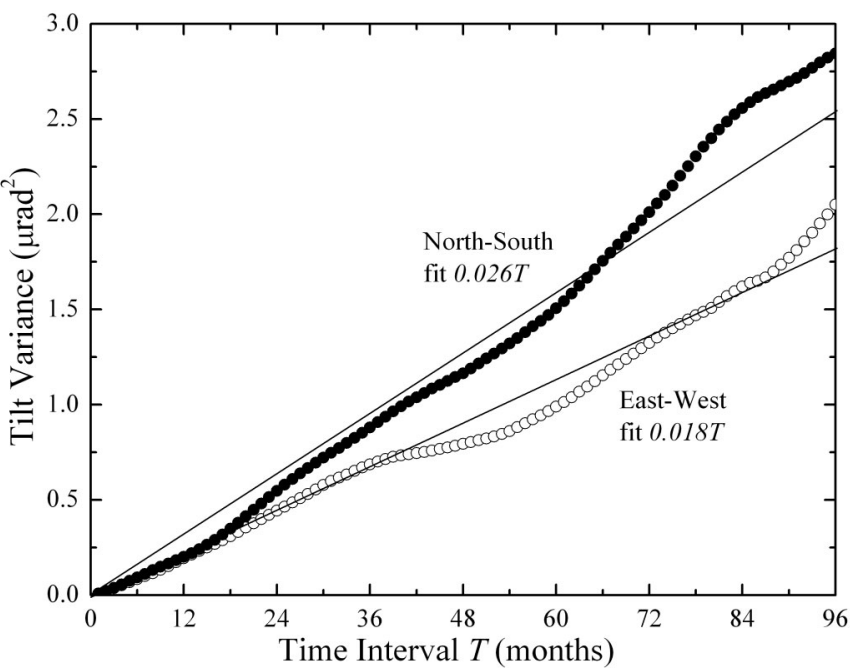

FIG. 28. Variance of the tilt elevation vs time interval (from Ref. [17]).

$0.018 \mu \mathrm{rad}^{2} / \mathrm{month}$ for the E-W tilt data (see dashed lines in Fig. 28).

The observed time dependence of the variance $\propto T$ is characteristic of a random walk (or Brownian) process. If one assumes the validity of the ATL law, then the diffusion coefficients can be estimated as $A_{\mathrm{ESNS}}=\left\langle d \Theta^{2}(T)\right\rangle L / T \approx$ $0.51 \times 10^{-6} \mu \mathrm{m}^{2} / \mathrm{s} / \mathrm{m}$ for the N-S tilt variations and $A_{\mathrm{ESEW}} \approx 0.35 \times 10^{-6} \mu \mathrm{m}^{2} / \mathrm{s} / \mathrm{m}$ for the E-W tilt drifts.

\section{b. Sazare mine and other tunnels}

A series of high precision ground motion measurements with several hydrostatic level systems has been performed by the group of Professor Shigeru Takeda of KEK (Japan) since the early 1990s. A 50-m-long HLS system with an overall accuracy of $0.1 \mu \mathrm{m}$ was used in an old Sazare mine (Sumitomo Metal Mining Co., Ltd., Shikoku, Japan) located about $300 \mathrm{~m}$ under the surface of a hard rock (green schist) mountain slope. The detected tilt was found to be a superposition diffusive of drifts, tides, and precipitation effects-see the PSD of the tilt observed in month long observations in 1993 in Fig. 29 from [40]. One can clearly see several tidal peaks in the spectrum. The straight line indicates the $1 / f^{2}$ dependence that corresponds to the ATL-law spectrum [Eq. (5)] with $A_{\text {Sazare }}=0.12 \times$ $10^{-6} \mu \mathrm{m}^{2} / \mathrm{s} / \mathrm{m}$. Significant seasonal variations were reported, too, with the diffusive having a maximum in December 1992 and a minimum in March 1993.

Similar studies with a 12 -m-long and a 42 -m-long watertube HLS system were carried out in the tunnel of the TRISTAN storage ring (KEK, Tsukuba, Japan) and it was found that the power spectral densities could be also approximated by Eq. (5) with a considerably bigger value of the diffusion coefficient $A_{\text {TRISTAN }_{\text {HLS }}} \approx 40 \times$ $10^{-6} \mu \mathrm{m}^{2} / \mathrm{s} / \mathrm{m}$ [41] - in a good agreement with the diffusion estimates obtained above from the TRISTAN orbit 


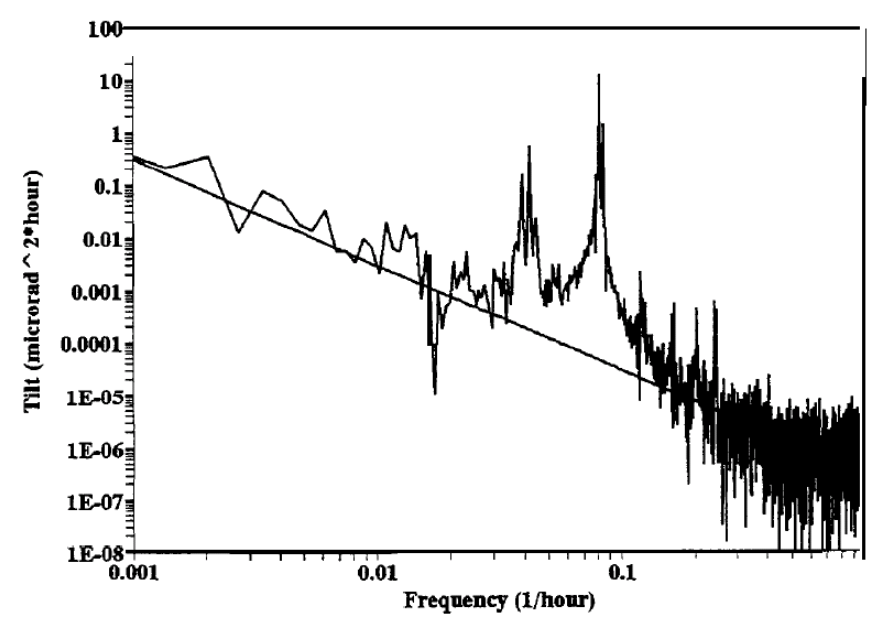

FIG. 29. A spectrum of ground motion in Sazare mine (Japan). The straight line indicates $1 / f^{2}$ (from Ref. [40]).

TABLE I. Summary of ground diffusion measurements.

\begin{tabular}{|c|c|c|c|c|c|c|}
\hline & & $A, 10^{-6} \mu \mathrm{m}^{2} / \mathrm{s} / \mathrm{m}$ & Time & Scale & Reference & Comments \\
\hline \multicolumn{7}{|c|}{ Beam orbit drifts in accelerators } \\
\hline HERA- $e$ vertical & $T$ & $4 \pm 2$ & 25 days & $6.3 \mathrm{~km}$ & {$[18]$} & $25 \mathrm{~m}$ deep, $\Delta L=23 \mathrm{~m}$ \\
\hline HERA- $p$ vertical & $T$ & $8 \pm 4$ & 5 days & $6.3 \mathrm{~km}$ & {$[17,18]$} & $25 \mathrm{~m}$ deep, $\Delta L=47 \mathrm{~m}$ \\
\hline TRISTAN vertical & $T$ & $27 \pm 7$ & 2 days & $3.0 \mathrm{~km}$ & {$[17,19]$} & $12 \mathrm{~m}$ deep, $\Delta L=47 \mathrm{~m}$ \\
\hline Circumference KEK-B & $T$ & $27 \pm 3$ & 4 months & $3.0 \mathrm{~km}$ & {$[20]$} & $\beta \approx 2.2$ \\
\hline Tevatron vertical & $T$ & $2.6 \pm 0.3$ & $15 \mathrm{hrs}$ & $6.3 \mathrm{~km}$ & [21] & $\sim 7 \mathrm{~m}$ deep, $\Delta L=30 \mathrm{~m}$ \\
\hline Horizontal & $T$ & $1.8 \pm 0.2$ & $15 \mathrm{hrs}$ & $6.3 \mathrm{~km}$ & & \\
\hline LEP vertical & $T$ & $10.9 \pm 6.8$ & $18 \mathrm{hrs}$ & $26.7 \mathrm{~km}$ & {$[22,24]$} & $\sim 100 \mathrm{~m}$ deep, $\Delta L=39 \mathrm{~m}$ \\
\hline LEP vertical & $T$ & $39 \pm 23$ & $3.3 \mathrm{hrs}$ & $26.7 \mathrm{~km}$ & {$[23]$} & Tides not excluded \\
\hline Horizontal & $T$ & $32 \pm 19$ & $3.3 \mathrm{hrs}$ & $26.7 \mathrm{~km}$ & [23] & Tides not excluded \\
\hline SPS vertical & $T$ & $6.3 \pm 3.0$ & $2 \mathrm{hr}$ & $6.9 \mathrm{~km}$ & [23] & $50 \mathrm{~m}$ deep, $\Delta L=32 \mathrm{~m}$ \\
\hline \multicolumn{7}{|c|}{ Accelerator Alignment Data Analysis } \\
\hline CERN LEP vertical & $L, T$ & $6.8-9.0$ & $6,9 \mathrm{mos}$ & $26.7 \mathrm{~km}$ & {$[17,26]$} & 45-170 m deep \\
\hline & & $3 \pm 0.6$ & 6 years & $26.7 \mathrm{~km}$ & [27] & $\Delta L=39 \mathrm{~m}$ \\
\hline CERN SPS vertical & $L, T$ & $14 \pm 5$ & $3-12 \mathrm{yr}$ & $6.9 \mathrm{~km}$ & [28] & $50 \mathrm{~m}$ deep, $\Delta L=32 \mathrm{~m}$ \\
\hline Tevatron vertical & $L, T$ & $4.9 \pm 0.1$ & $1-6 \mathrm{yr}$ & $6.3 \mathrm{~km}$ & {$[29,30]$} & $\sim 7 \mathrm{~m}$ deep, $\Delta L=30 \mathrm{~m}$ \\
\hline SLAC PEP vertical & $L$ & $100 \pm 50$ & $20 \operatorname{mos}$ & $2 \mathrm{~km}$ & {$[28]$} & Cut-and-cover tunnel \\
\hline SLAC linac vertical & $L$ & $200 \pm 100$ & $17 \mathrm{yr}$ & $3 \mathrm{~km}$ & {$[15,28]$} & Cut-and-cover tunnel \\
\hline & $L$ & $<10$ & $17 \mathrm{yr}$ & $3 \mathrm{~km}$ & {$[31]$} & Linear trends removed \\
\hline UNK site vertical & $L$ & $100 \pm 50$ & $2 \mathrm{yr}$ & $500 \mathrm{~m}$ & {$[15,28]$} & Surface monuments \\
\hline & & \multicolumn{5}{|c|}{ Geophysics Instruments Data } \\
\hline PFO (CA, USA) & $T$ & 0.7 & $5 \mathrm{yr}$ & $732 \mathrm{~m}$ & {$[32]$} & Laser interferometer \\
\hline SLAC linac vertical & $T$ & $1.4 \pm 0.2$ & $0.5 \mathrm{hr}$ & $3 \mathrm{~km}$ & [34] & $\Delta L=1500 \mathrm{~m}$ \\
\hline & $T$ & $0.2-2$ & $1 \mathrm{hr}$ & $3 \mathrm{~km}$ & [35] & From PSD fit \\
\hline CERN PS pillar & $T$ & $3 \pm 1$ & $2.5 \mathrm{yr}$ & $10 \mathrm{~m}$ & {$[36,37]$} & $10 \mathrm{~m}$ depth \\
\hline SLAC FFTB & $T$ & $0.1-0.5$ & $15 \mathrm{hrs}$ & $\sim 30 \mathrm{~m}$ & {$[38]$} & Wire, in the lab \\
\hline Esashi (Japan) & $T$ & $0.3-0.5$ & 15 years & $50 \mathrm{~m}$ & {$[17,39]$} & $60 \mathrm{~m}$ deep, NS-EW \\
\hline Sazare (Japan) & $T$ & $0.01-0.12$ & 6 weeks & $48 \mathrm{~m}$ & {$[40]$} & $300 \mathrm{~m}$ deep \\
\hline Kamaishi (Japan) & $T$ & $0.06-0.14$ & & & [43] & Granite \\
\hline SPring8 (Japan) & $T$ & 0.8 & & & [43] & Granite \\
\hline Miyazaki (Japan) & $T$ & 15 & & & [43] & Diorite \\
\hline Rokkoh (Japan) & $T$ & $\sim 36$ & & & [43] & Granite \\
\hline KEK-B tunnel & $T$ & 40 & 4 days & $42 \mathrm{~m}$ & [41] & $12 \mathrm{~m}$ deep, joints \\
\hline FNAL PW7 & $T$ & $6.4 \pm 3.6$ & 3 months & $180 \mathrm{~m}$ & [45] & $\Delta L=30 \mathrm{~m}, t^{\circ}$-effects \\
\hline FNAL MI8 line & $T, L$ & $1-10$ & 1 month & $285 \mathrm{~m}$ & [46] & $\Delta L=15$ m, m.b. $\gamma<1$ \\
\hline FNAL Tevatron & $T, L$ & $2.2 \pm 1.2$ & 1 week & $600 \mathrm{~m}$ & {$[30]$} & $\Delta L=30 \mathrm{~m}, \gamma=0 L>120 \mathrm{~m}$ \\
\hline FNAL MINOS hall & $T, L$ & 0.18 & 1 month & $90 \mathrm{~m}$ & {$[30]$} & $\Delta L=30 \mathrm{~m}, \sim 100 \mathrm{~m}$ deep \\
\hline Aurora mine (IL) & $T, L$ & $0.6 \pm 0.3$ & 2 weeks & $210 \mathrm{~m}$ & {$[46,47]$} & $\Delta L=30 \mathrm{~m}, \sim 100 \mathrm{~m}$ deep \\
\hline
\end{tabular}


motion. It was noted that the largest relative motion takes place across the different tunnel blocks separated by expansion joints.

The diffusion studies in several more tunnels in Japan confirmed that the ATL-law scaling [Eq. (5)] offers a very good fit to most of the data, and concluded that the diffusion parameter $A$ is influenced dominantly by the earth and rock properties $[42,43]$. The observed parameter $A$ is smaller in the tunnel in a solid rock than in the broken rock. The excavation method of the tunnel also affects significantly the diffusion: e.g., a tunnel made by dynamite blasting had $A=5 \times 10^{-6} \mu \mathrm{m}^{2} / \mathrm{s} / \mathrm{m}$ while a tunnel in a similar rock bored by a tunnel-boring-machine had $A=$ $1 \times 10^{-6} \mu \mathrm{m}^{2} / \mathrm{s} / \mathrm{m}$. Such a difference was attributed to artificial fragmentation of the rock during the construction. Values of the diffusion coefficients measured in various Japanese tunnels will be presented in Table I below.

\section{HLS measurements in Luxembourg}

The power-law ground drifts have also been observed in the measurements with a 43-m-long floatless water-tube tiltmeter which has been in operation since 1997 at the Walferdange Underground Laboratory for Geodynamics in the Grand Duchy of Luxembourg [44]. The instrument's very low noise level and its high resolution up to the longperiod seismic band (where for instance the resolution is better than $5 \times 10^{-12} \mathrm{rad}$ ) allow the successful recording of minuscule drifts as well as rarely observed grave toroidal and spheroidal free oscillations of the Earth excited by major earthquakes. In the environmental conditions of its installation (in a gypsum mine at $100 \mathrm{~m}$ depth), the instrument shows a high degree of reliability and a very low drift rate $(<0.005$ microradian/month). The observed spectrum of the tilt is shown in Fig. 30 and has distinct power-law scaling at frequencies below $0.0001 \mathrm{~Hz}$ PSD $\propto$

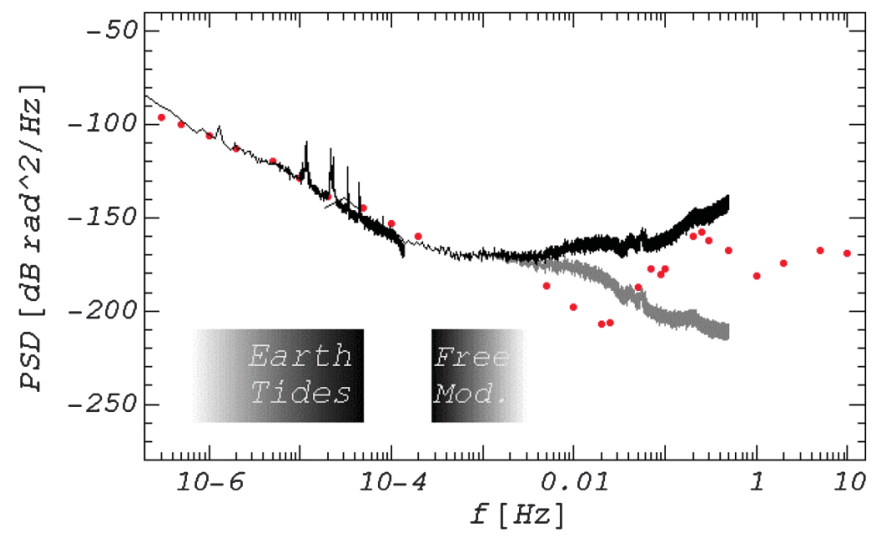

FIG. 30. (Color) The PSD of the 6-years-long record in Walferdange (with and without the instrumental response correction-black and gray curve, respectively) and the low tilt noise reference model from Ref. [9] (red dots). Shaded rectangles pinpoint the frequencies ranges of the Earth tides and the Earth free modes (from Ref. [44]).
$1 / f^{2.2}$ (red dots); an effective ATL-diffusion constant at the lowest frequency of $f=2 \times 10^{-7} \mathrm{~Hz}$ can be found from Eq. (5) to be about $A=0.1 \times 10^{-6} \mu \mathrm{m}^{2} / \mathrm{s} / \mathrm{m}$.

\section{Measurements with multiprobe HLS systems in Illinois}

The HLS measurements reviewed above are examples of the ground diffusion in time as in all of them only two HLSs were used. To observe the diffusion in the space domain, a series of extensive ground motion studies with many interconnected HLS probes has been performed in various locations in Illinois. High precision HLS probes developed for these studies (see Fig. 31) are capacitive sensors equipped with local water temperature meters needed for thermal expansion compensation. The probes are made in two configurations - one for use with a single 1 inch diameter half-filled water pipe, and another for use with two separate $\frac{1}{2}$ inch diameter tubes for air and for water (fully filled).

A pair of the probes set side by side shows the differential noise level of $\sigma^{2}=(0.09 \mu \mathrm{m})^{2}+1.252 \times$ $10^{-7} \mu \mathrm{m}^{2} / \mathrm{s} \mathrm{T}$ (more details can be found in Ref. [48]). In a typical measurement arrangement, six to 20 of such probes were installed in the same water level system spaced 15 to 30 meters apart (usually along the line as shown in Fig. 32). Once a minute, a PC based data acquisition system collects the water level data (averaged over the minute). The temperature readings of all the probes are being recorded simultaneously and later used for correction, as well as the readings from one or two air pressure monitors.

\section{a. Proton West tunnel at FNAL}

Studies in the Proton West (PW) tunnel on the site of the Fermi National Accelerator Laboratory had been carried out in 1999-2000 [45]. The PW is an unused beam line for fixed target experiments with a shallow ( $\sim 5 \mathrm{~m}$ depth) tunnel built by the "cut-and-cover" method in the 1970s. It has a flat concrete floor that made quite easy the installation of six HLSs over a total length of $180 \mathrm{~m} \mathrm{(30+30+}$ $60+30+30$ meter apart).

An important drawback of the tunnel was that it was not sealed and there were large temperature variations from one end to the other sometimes by a few ${ }^{\circ} \mathrm{C}$ a day causing large changes in the water level readings-see Fig. 33. The ground tilts due to earth tides occurred twice a day with some $20 \mu \mathrm{m}$ peak-to-peak amplitude in the level difference $Y_{2}-Y_{6}$ between probes \#2 and \#6 $150 \mathrm{~m}$ apart. Notably, the tides are practically unseen in the second difference $\mathrm{SD}_{2446}=Y_{2}-2 Y_{4}+Y_{6}$. The variance of the second difference grows approximately linear with the time interval $\left\langle\mathrm{SD}_{2446}{ }^{2}(T)\right\rangle \approx T \times 114 \mu \mathrm{m}^{2} /$ day $\quad$ (see dashed line in the bottom plot in Fig. 33). Making statistical analysis for all possible combination of probes, one gets the ATL-law diffusion coefficient of about $A_{\mathrm{PW}}=$ $(6.4 \pm 3.6) \times 10^{-6} \mu \mathrm{m}^{2} / \mathrm{s} / \mathrm{m}$. The lack of data points in 

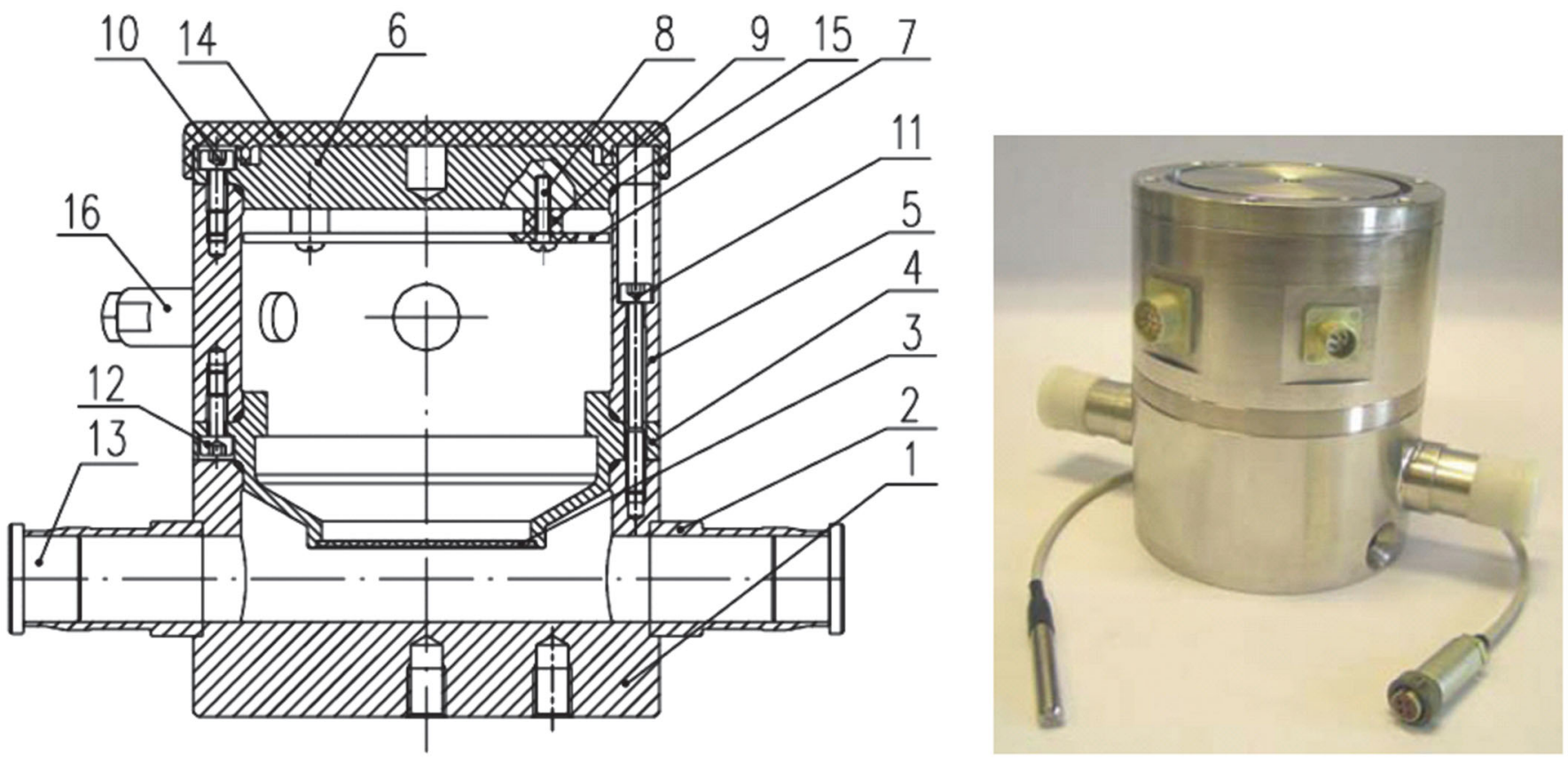

FIG. 31. (Color) SAS-2 HLS sensor used in the ground motion studies in Illinois (from Ref. [48]).

spatial intervals does not allow one to confirm or to reject the $L$ dependence of the variance.

\section{b. MI8 tunnel at FNAL}

Ground motion studies in the MI8 (Main Injector $8 \mathrm{GeV}$ ) tunnel took place over a few months in 20022003 and employed 20 HLS sensors equidistantly installed over 285-m-long line (so, the probe-to-probe distance was $15 \mathrm{~m}$ ) [46]. The tunnel is shallow and of a similar construction type and geology as the PW tunnel and the Tevatron tunnel discussed above. For several months the observed water levels data were dominated by a quasiperiodic motion with an amplitude of about $10 \mu \mathrm{m}$ every $\sim 2$ hours. Finally, the source was tracked to a domestic water well located $219 \mathrm{ft}$ deep and several hundred feet away from the MI8 tunnel which slowly and periodically changed the ground water level. At the end, only one

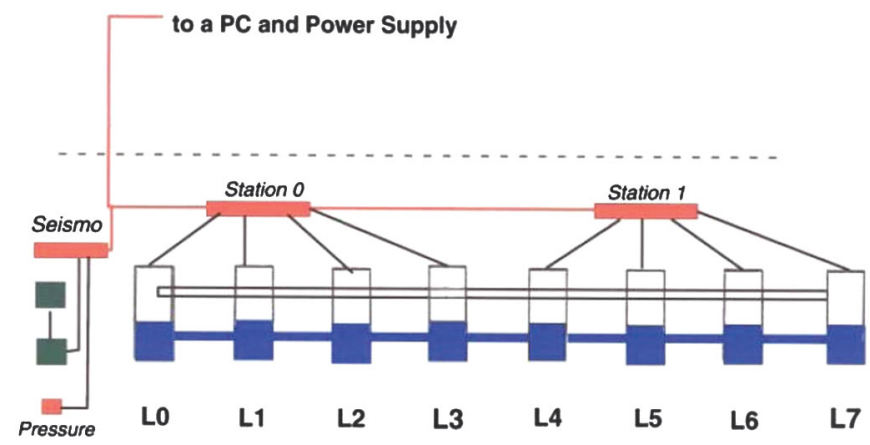

FIG. 32. (Color) Schematics of the systems of HLS sensor used in the studies in Illinois. month of February 2003 was available for low-noise measurements of the ground diffusion. The coefficients $A$ calculated as $A=\left\langle\mathrm{SD}_{n m m l}{ }^{2}(T)\right\rangle / T / 2 L$, where the indexes $(n, m, l)$ indicate triples of the sensors distanced by $L$ and

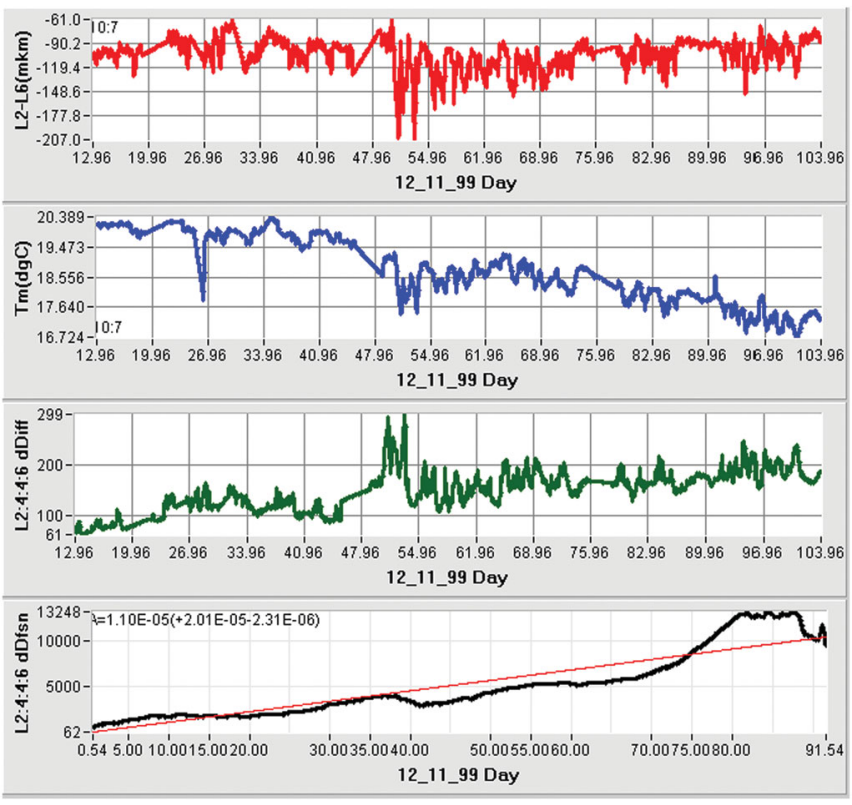

FIG. 33. (Color) 91 days data records starting November 12, 1999 from the PW studies: (top to bottom) the level difference between probes \#2 and \#6 120 (vertical scale of about $150 \mu \mathrm{m}$ ), mean temperature in the tunnel (vertical scale of $3.5^{\circ} \mathrm{C}$ ), the second level difference $\mathrm{SD}_{2446}$ (see in the text, scale $240 \mu \mathrm{m}$ ), and variance of the second level difference $\mathrm{SD}_{2446}$ for intervals of up to 91 days (from Ref. [45]). 


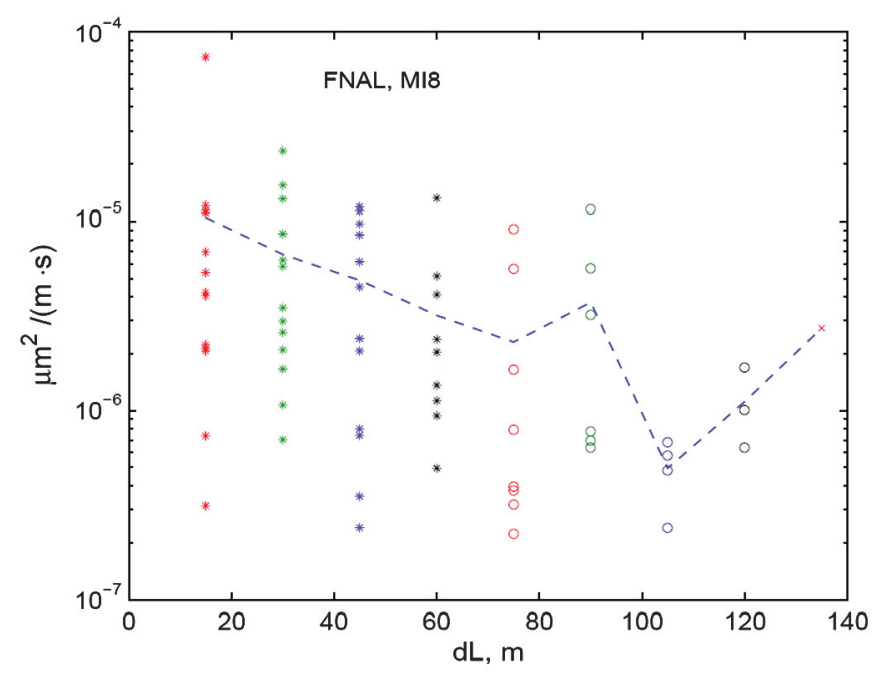

FIG. 34. (Color) Diffusion coefficient $A$ calculated for all possible combination of the probes distanced by $L$ from 15 to $135 \mathrm{~m}$ from 1 month data records in MI8 tunnel [46].

$T=1$ month, are shown in Fig. 34. For example, the circles at $L=120 \mathrm{~m}$ data are for three combinations of the sensors (\#1, \#9, \#17), (\#2, \#10, \#18), and (\#3, \#11, \#19). One can see that the range of the $A$ 's covers the PW results and is roughly constant for distances $L$ from 15 to $90 \mathrm{~m}$. However, the mean value of $A_{M I 8}=(1-10) \times$ $10^{-6} \mu \mathrm{m}^{2} / \mathrm{s} / \mathrm{m}$ appears to decrease slightly with $L$, as if the variance scales as $d Y^{2} \propto T^{1} L^{\gamma}$ with $0<\gamma<1$.

\section{c. Tevatron tunnel at FNAL}

Since early 2004, a system of 20 HLS sensors with halffilled water pipe was installed in the Tevatron tunnel on top of the accelerator focusing magnets spaced $30 \mathrm{~m}$ apartsee Fig. 35.

The environment of a working accelerator had its own peculiarities, e.g., regular ramping of the electromagnets resulted in few micron relative magnet position changes-

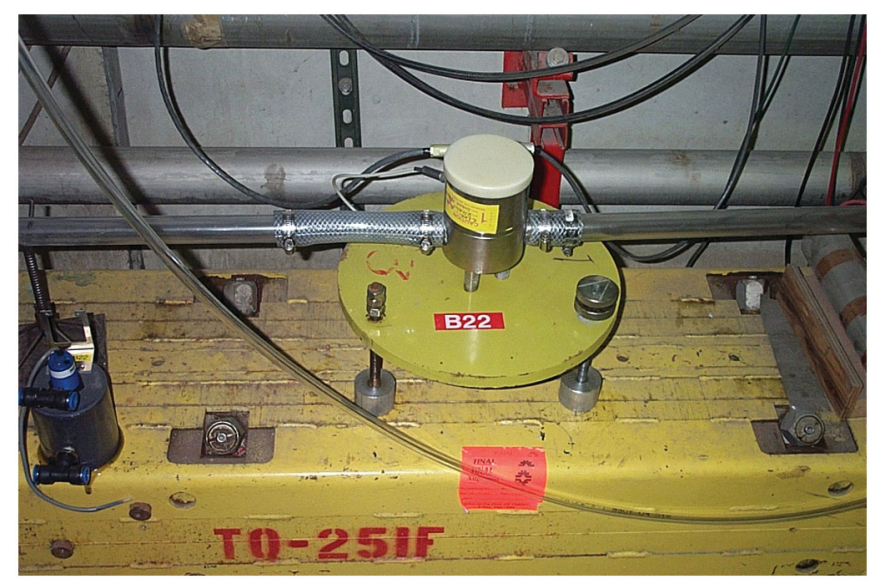

FIG. 35. (Color) HLS probe on Tevatron accelerator focusing magnet.

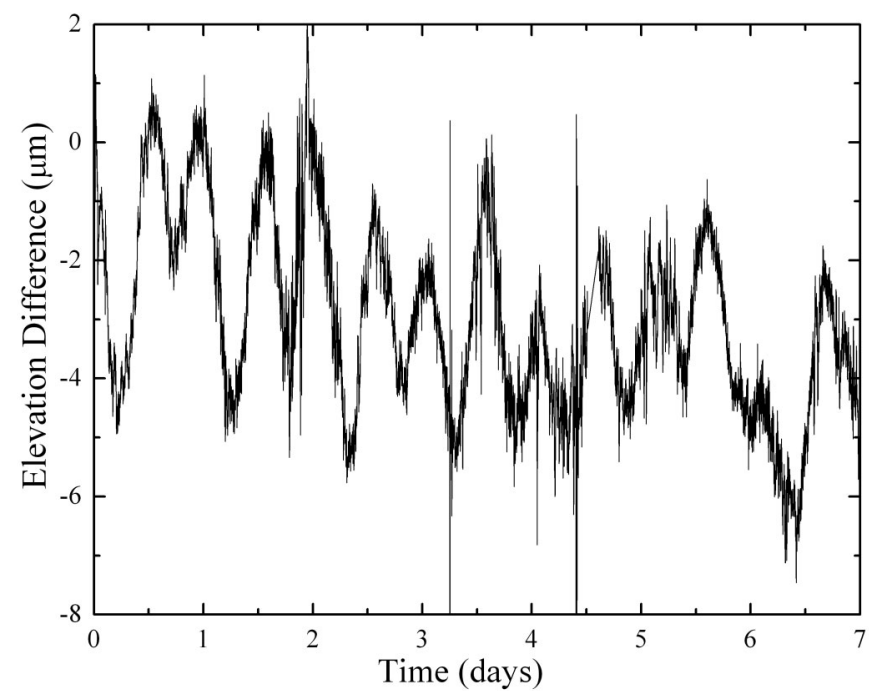

FIG. 36. One week record of elevation difference of two neighbor focusing magnets in the Tevatron tunnel as measured by HLS (starts midnight February 7, 2004; Ref. [30]).

see spikes in Fig. 36 from Ref. [30]—on top of regular tidal variations and diffusive drifts. Figure 37 shows a snapshot of the magnet elevation changes after 23 days of observations. One can see that the differential movements over the $\sim 600 \mathrm{~m}$ section of tunnel could be as big as $30-50 \mu \mathrm{m}$.

Variograms of the second differences have been analyzed, linear dependence on the time interval $T$ confirmed, and the variance $\left\langle\mathrm{SD}_{n m m l}{ }^{2}(T)\right\rangle / T$ is plotted in Fig. 38. As in the MI8 tunnel data analysis, the indices $(n, m, l)$ indicate triples of the sensors distanced by $L$ and $T=7$ days-the week of February 7, 2004. One can see that the variance increases with $L$ up to $90-120 \mathrm{~m}$ and then flattens out. That indicates lack of coherence (independence) of the motion

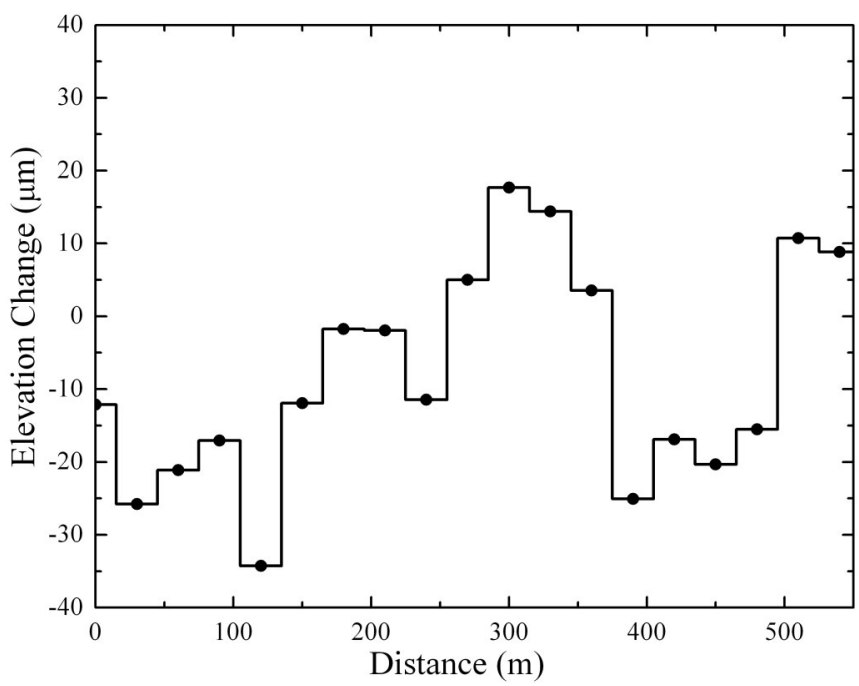

FIG. 37. Change of the elevations of 20 Tevatron magnets after 23 days of observations (January 7-February 1, 2004; from Ref. [30]). 


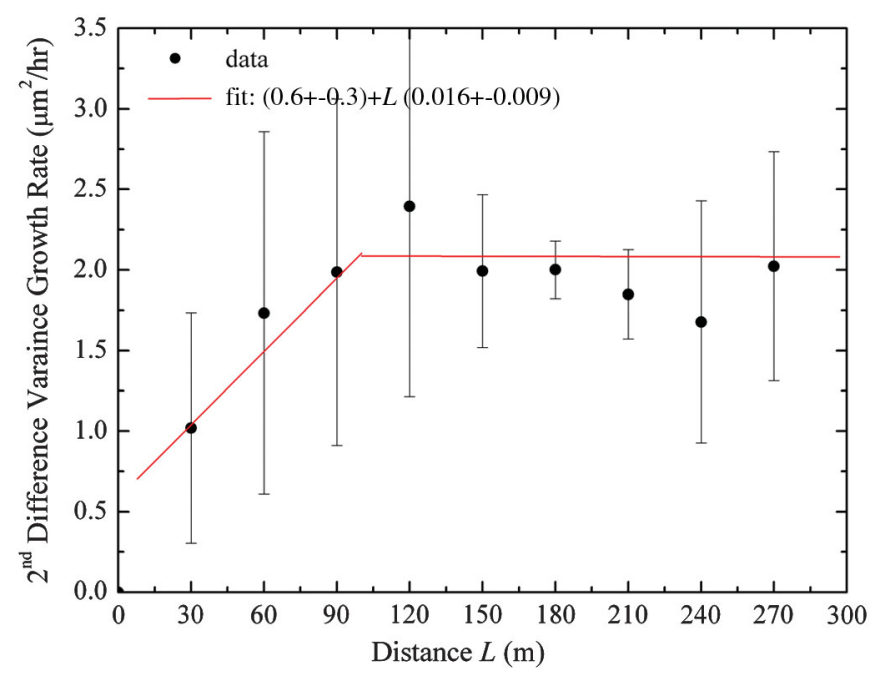

FIG. 38. (Color) Dependence of the growth rate of the variance of the 2nd difference vs distance between the HLS probes in the Tevatron tunnel, the week of February 7, 2004 (from Ref. [30]).

of the pieces of the tunnel distanced by more than $120 \mathrm{~m}$ apart-at the time scale of 1 week. For shorter distances, the ATL law with coefficient $A_{\mathrm{Tev} B}=(2.2 \pm 1.2) \times$ $10^{-6} \mu \mathrm{m}^{2} / \mathrm{s} / \mathrm{m}$ gives a good approximation of the data. This diffusion coefficient is close to the one estimated from the accelerator beam orbit motion discussed above in Sec. II.

\section{d. MINOS experiment hall at FNAL}

Seven HLS probes had been installed in 2006 in the MINOS experiment underground hall some 100 meters below grade on top of the Galena Platteville dolomite (also on the site of Fermilab). The probes are set $30 \mathrm{~m}$ apart and connected in two double-pipe (air/water) systems - the first one with four probes is orientated along a

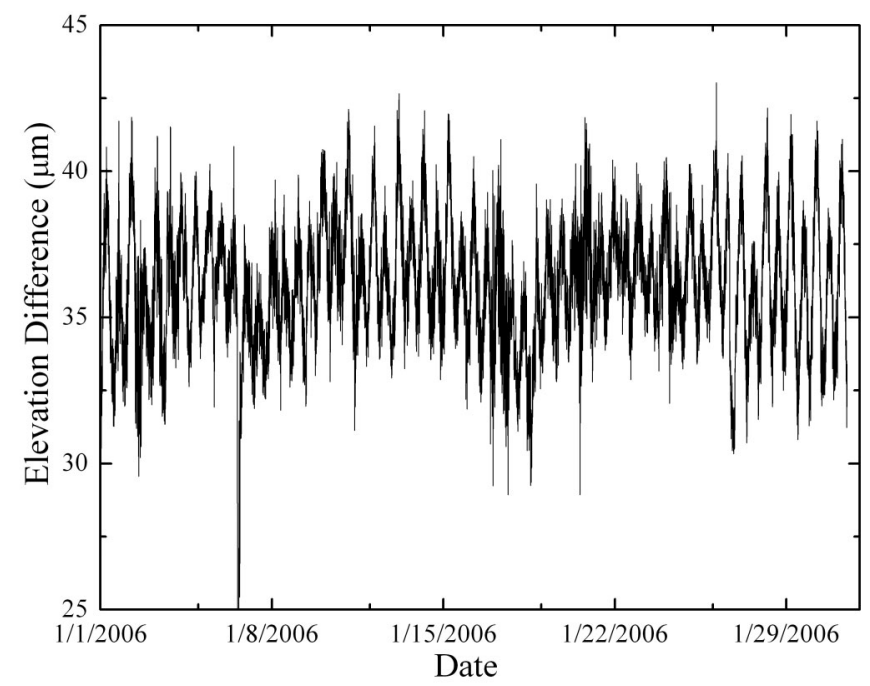

FIG. 39. January 2006 record of elevation difference for two HLS probes $90 \mathrm{~m}$ apart in the FNAL MINOS hall [30].

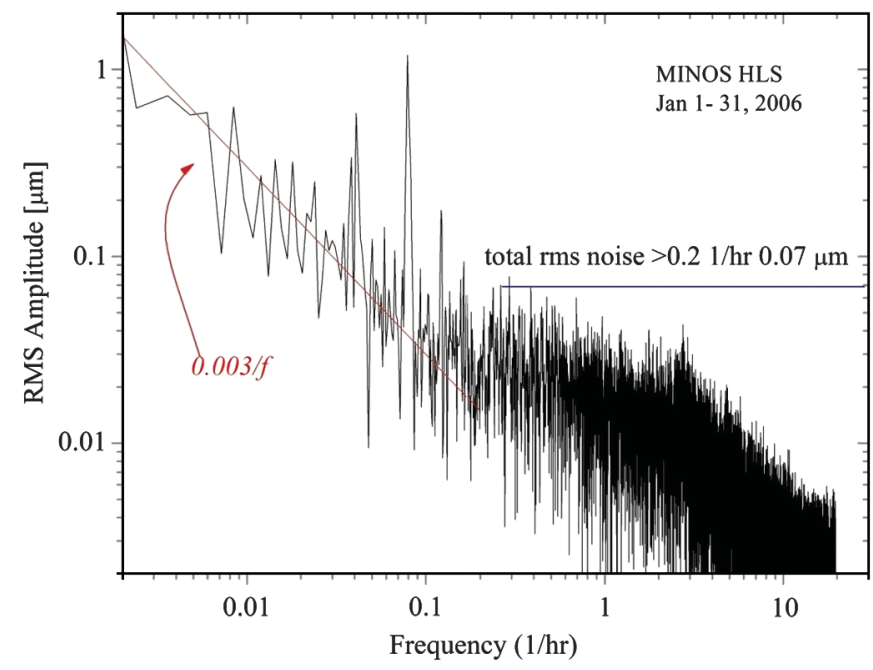

FIG. 40. (Color) FFT of the elevation difference for HLS probes $90 \mathrm{~m}$ apart as measured in the Fermilab's MINOS hall [30].

North-South line and the other system of three oriented along an East-West line. One month-long record of the HLS readings of the level difference $Y_{0}-Y_{3}$ (probes \#0 and \#3, $90 \mathrm{~m}$ apart in the N-S direction) is presented in Fig. 39. One can see that some $6 \mu \mathrm{m}$ amplitude periodic variations due to the Earth tide dominate few $\mu \mathrm{m}$ scale slow drifts over weeks.

To remove the systematic effects due to the tides, the gast Fourier transformation (FFT) of the one-month-long record of the level difference $Y_{0}-Y_{3}$ data has been calculated (see Fig. 40). The power-law fit $1 / f$ indicated by the red line in Fig. 40 corresponds to the ATL-diffusion coefficient of $A_{\text {MINOS }}=0.18 \times 10^{-6} \mu \mathrm{m}^{2} / \mathrm{s} / \mathrm{m} \mathrm{[30]}$.

\section{e. Aurora mine}

Since early 2000, continuous slow ground motion measurements with up to eight HLS probes are being carried out in a $100 \mathrm{~m}$ deep dolomite mine (Conco-Western Co./ LaFarge Co., North Aurora, IL)—some 3 miles SouthWest of Fermilab. This is a multilayer mine in GalenaPlattville dolomite. Our 210-m-long system was set at the depth of about $80 \mathrm{~m}$ near the border wall of this $0.8 \mathrm{~km} \times$ $1.4 \mathrm{~km}$ underground facility. During the studies the mine continued dolomite production and some 3 tons of explosives were detonated each day at around 3 p.m., except weekends in different areas and at different levels of the mine. A ventilation system makes the temperature of the mine very dependent on the outside temperature.

Figure 41 shows one month data records in the Aurora mine in January 2000. The horizontal axis is time in days in December 2000 (e.g., 31.96 correspond to late night of December 31, 2000). The vertical axis on Fig. 41(a) is for a relative vertical position of two observation points 180 meters apart (total scale is $895-813=82 \mu \mathrm{m}$ ). Because of periodic changes in relative positions in the system Moon- 


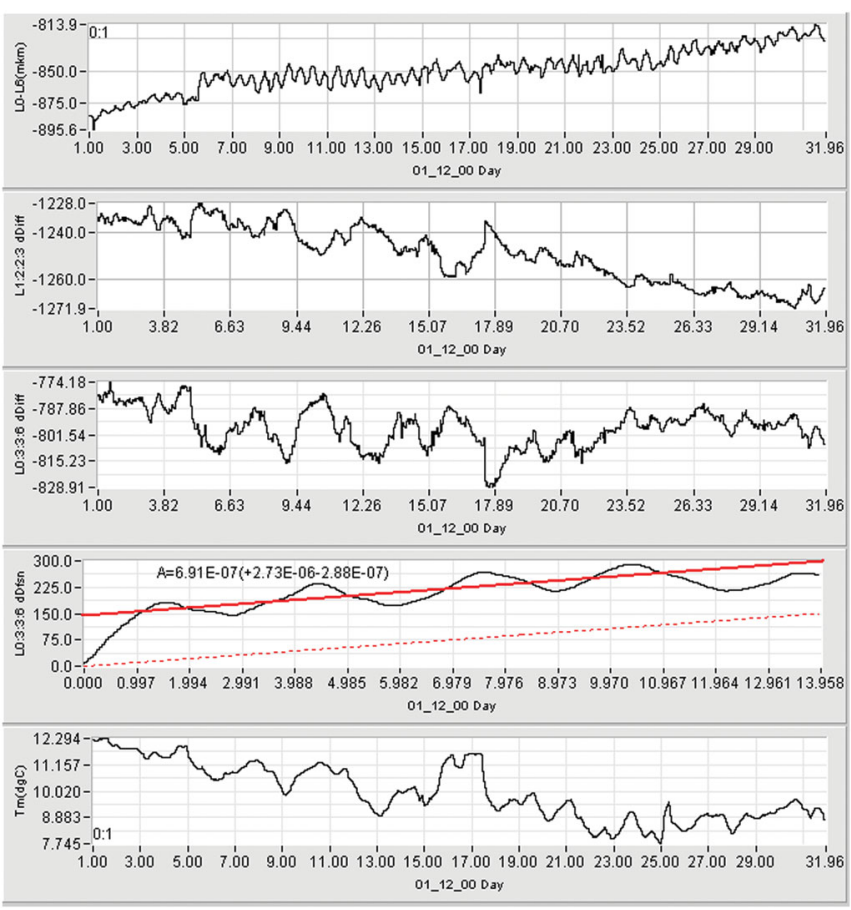

FIG. 41. (Color) Slow ground motion in $120 \mathrm{~m}$ deep dolomite mine (Aurora, IL) in December 2000. Top to bottom (a) to (e), see comments in the text [47].

Earth-Sun, the amplitude of diurnal oscillations varies with a period of 14 days - it is obviously less at the beginning of the plot and in the middle of the month. Obvious creep (slow change of the tilt) of the order of $82 \mu \mathrm{m} / 180$ meters $=0.5 \mu \mathrm{rad}$ is seen over one month in the same plot. Possible explanations for this change are natural geological instability, temperature effect, or atmospheric pressure effect. Figure $41(\mathrm{e})$ reveals $1^{\circ} \mathrm{C}$ variations in the Aurora mine daily and some $4^{\circ} \mathrm{C}$ drop in the temperature over 3 weeks. To separate the temperature effects and the ground diffusion from the tides, the second difference $\mathrm{SD}_{1223}$ for the probes $30 \mathrm{~m}$ apart $\mathrm{SD}_{0336}$ for the probes $90 \mathrm{~m}$ apart are computed and plotted in Figs. 41(b) and 41(c). One can see that they are correlated with the average temperature changes with coefficients about $-20 \mu \mathrm{m} /{ }^{\circ} \mathrm{C}$ and $+40 \mu \mathrm{m} /{ }^{\circ} \mathrm{C}$ correspondingly. Air pressure also can contribute into the motion of the ground, both in $\mathrm{SD}_{1223}$ and $\mathrm{SD}_{0336}$ but it is usually prominent only over longer distances of $L>1 \mathrm{~km}$. Besides regular Earth tides and temperature drifts, the ground does move randomly due to the natural diffusion. Figure 41(d) shows the mean square of the second vertical difference for the points 90 meters apart, and the red line presents linear fit $\left\langle\mathrm{SD}_{0336}{ }^{2}(T)\right\rangle=150+2 \mathrm{ATL}$, with $A=0.69 \times$ $10^{-6} \mu \mathrm{m}^{2} / \mathrm{s} / \mathrm{m}$ and $T$ up to 14 days. Somewhat excessive motion at short periods $T<1$ day can be explained by the ground jumps due to the daily blasts taking place in the mine (within 1 mile from the measurement system location) — several of them with amplitudes of 10 to 25 microns

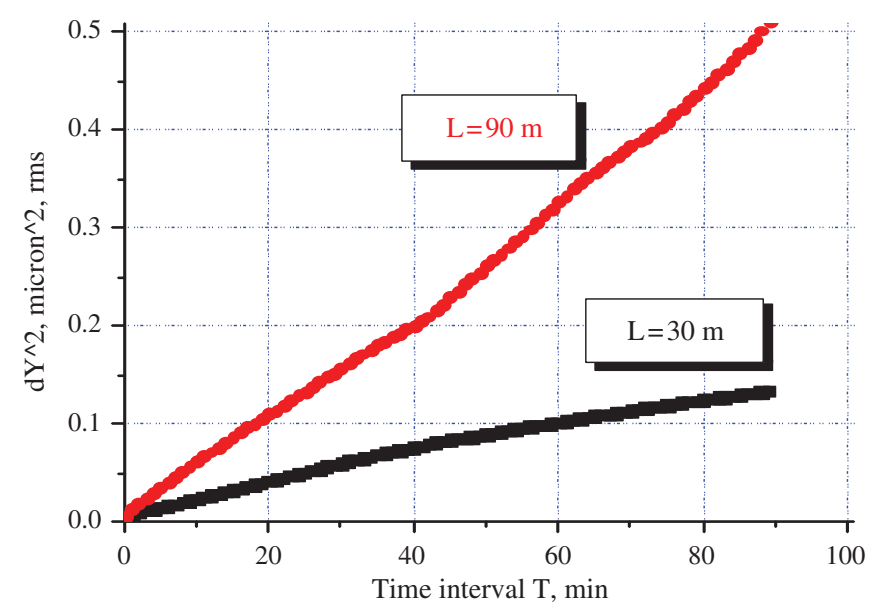

FIG. 42. (Color) Variance of the vertical relative ground motion for the points 30 and $90 \mathrm{~m}$ apart, measured on October 13-15, 2000 in the Aurora mine in Illinois [46].

are seen in Fig. 41(a). Extraction of temperature correlated components and linear drifts leads to the average (over all combination of the second differences and over all possible $L=30,60,90 \mathrm{~m})$ value of $A_{\text {Aurora }}=(0.58 \pm 0.28) \times$ $10^{-6} \mu \mathrm{m}^{2} / \mathrm{s} / \mathrm{m}$.

There were no blasts over weekends as well as sometimes the temperature does not change much as well, so one can use such records for analyzing "natural" ground diffusion at shorter time scales. For example, on a quiet weekend of October 13-15, 2000, the temperature variation was less than $0.05^{\circ} \mathrm{C}$. The 2 days record analysis is presented in Fig. 42 which depicts the variance of the second differences $\left\langle\mathrm{SD}_{1447}{ }^{2}(T)\right\rangle(L=90 \mathrm{~m}$, red circles $)$ and $\left\langle\mathrm{SD}_{1223}{ }^{2}(T)\right\rangle(L=30 \mathrm{~m}$, black squares) for the time intervals of up to $T=90$ minutes. In good accordance with the ATL law, the variances grow linearly with $T$, the variance is about 3 times larger for a 3 time larger distance, and corresponding diffusion coefficients are almost the same $A_{90}=0.53 \times 10^{-6} \mu \mathrm{m}^{2} / \mathrm{s} / \mathrm{m}$ and $A_{30}=$ $0.42 \times 10^{-6} \mu \mathrm{m}^{2} / \mathrm{s} / \mathrm{m}$.

\section{DISCUSSION ON FRACTAL NATURE OF THE GROUND DIFFUSION}

\section{A. Discussion of the results}

Several conclusions can be made from the results presented above. First of all, the diffusive motion of the ground is typically just a background to much more powerful processes, like ground expansion due to temperature changes, or bending due to atmospheric pressure variation or winds, long-term settlement drifts or Earth tides. Special data processing is needed to separate diffusive noise from systematic or periodic signals: in the time or space domains, that can be achieved with use of digital filters, like the first or the second difference methods employed above; in the frequency or wavelength domains, Fourier analysis 
of windowed data sets (e.g., with a Hanning window) makes visible the power-law component of the spectrum.

Table I below summarizes the observations of the ground diffusion presented above and presents the diffusion coefficient $A$, the time interval $T$ of the observation or analysis, the spatial scale $L$ (e.g., the tunnel length, or the total length of the HLS system), plane ( $V$ is for vertical, $H$ is for horizontal), and effective depth of the observations. The second column indicates whether temporal $(T)$ or spatial $(L)$ characteristics of the diffusive ground motion have been explored. One can see that most of the accelerator orbit drift data and most of the HLS and laser interferometer studies reveal the diffusion in time. Many accelerator alignment data manifest the diffusion in space. Diffusion in both time and space is observed in many-year accelerator alignment data and in long-term measurements with HLS systems employing many (up to 20) probes.

Another conclusion which can be made is that the speed of the diffusion, the coefficient $A$, is site dependent and despite very large spread, it has some tendency of being smaller at bigger depths-as one can see in Fig. 43, also in harder rocks and in geologically stable locations (like those where geophysical observatories are set). Japanese data indicate that even the tunneling method may affect the diffusion rate. Taking the geometric mean of the data from the operational accelerator facilities, one gets $A_{\text {avg.accel. }}=10^{-5.15 \pm 0.5} \mu \mathrm{m}^{2} /(\mathrm{s} \mathrm{m})$ or approximately $7 \times$ $10^{-6} \mu \mathrm{m}^{2} / \mathrm{s} / \mathrm{m}$ - see dashed line in Fig. 43.

One can also notice that the ATL approximation is not always the best, and in general, the exponents in the fit $\left\langle d Y^{2}(T, L)\right\rangle \propto T^{\alpha} L^{\gamma}$ can significantly differ from 1 . Naturally, for small time intervals $T$ the movements of the ground elements are fully uncorrelated if they are separated by a long enough distance $L>L_{m}$-for example, by more than $120 \mathrm{~m}$ for 1 week intervals as seen

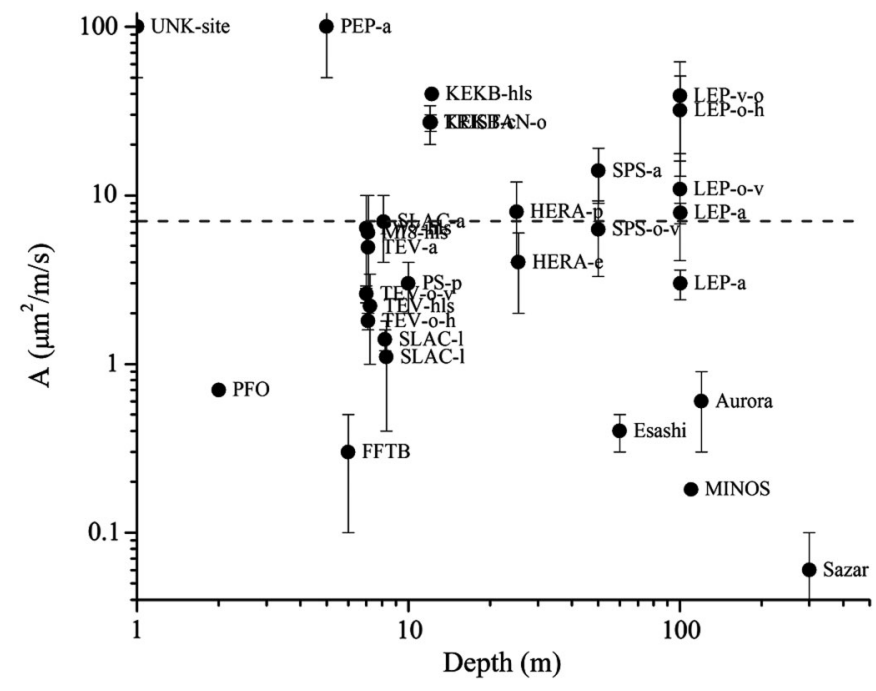

FIG. 43. Coefficients of the ground diffusion from Table I vs the depth of the observations. The dashed line represents the average value for operational accelerators. in the Tevatron HLS data on Fig. 38 or by more than $800 \mathrm{~m}$ for $1 \mathrm{yr}$ intervals as seen in the Tevatron alignment data discussed above in Sec. II C and presented in Fig. 18. On the basis of these two observations, one can suppose that the boundary scales approximately as $L_{m} \propto T^{1 / 2}$. Of course, with such a limited number of data sets, we could not explore in detail the boundary $L_{m}(T)$ beyond which the independence (or significant loss of correlation) occurs while it is a very important phenomena [49] which can shed light on the dynamics of the ground fractures.

The observations reviewed above cover time intervals from hours to several years and spatial scales from a dozen meters to a dozen of kilometers (the largest accelerators). There is some evidence of the diffusion at much larger $T$ or $L$ intervals. For example, 50 years observation (19301980) of sea levels in 12 Japanese ports distanced by as much as $800 \mathrm{~km}$ [50] showed that besides daily and seasonal changes, the level variation has a long-term "random walk" component $\left\langle d Y^{2}(T)\right\rangle \propto T$ with computed diffusion coefficient $A$ of about $35 \times 10^{-6} \mu \mathrm{m}^{2} / \mathrm{s} / \mathrm{m}$ [17]. It is long known to geophysicists that Earth's topography is fractal, and its power spectral density scales with the wave number as $S(k) \propto k^{-2}$ that corresponds to $\left\langle d Y^{2}(L)\right\rangle \propto L$ over distances 100 to $6000 \mathrm{~km}$ (see, e.g., Figs. 17 and 19 in Ref. [14] and the corresponding discussion). What our research adds to the previously known results is the notion that the diffusion takes place both in time and in space (at least, over the scales indicated in Table I and characteristic for high energy physics accelerators).

\section{B. Modeling diffusive ground motion}

The fractal objects and time series are one of the favorite subjects for modern studies on geophysics, geomorphology, hydrology, landscape evolution, etc., and a variety of models have been proposed and studied in great detail (see, e.g., [12-14,51] and references therein). To reproduce the "ATL law"-like motion in computer codes for accelerator design, several algorithms that produce the required space and time dependencies have been developed. In the case of a linear system (points of the ground are equally distributed along a straight line), it could be straightforward to apply the "random walk" procedure: for a given time step $k$ it is only necessary to start at one end, giving each point a random displacement $\Delta_{m}{ }^{k}$ with respect to the previous point $Y_{i}^{k}=Y_{i}^{k-1}+\sum_{m=0}^{i} \Delta_{m}{ }^{k}[6,18]$. It is easy to see that the variance of the resulting relative displacement of any two points separated by $L$ is given by ATL law [Eq. (4)]. With a bit more cumbersome mathematics, the method can be extended to any one-dimensional geometry shape (e.g., circle) on a two-dimensional surface [52].

Below we present a simple one-dimensional model of the landscape evolution which has a certain physical meaning, satisfies the ATL law, and produces a reduced correlation of the surface motion at large distances. The model is qualitatively similar to the one previously discussed in [53] 


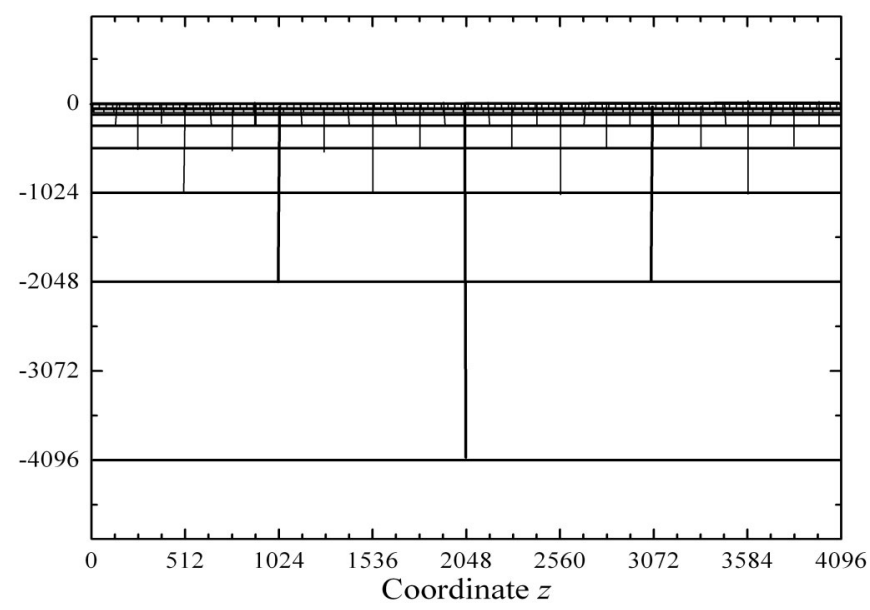

FIG. 44. Fractal set of ground blocks (see text).

and considers the ground as a set of separated blocks with different characteristic sizes $R$-as approximately shown in Fig. 44. The number of blocks $N_{b}(L, R)$ under any area of the scale $L$ scales with $R$ as $N_{b}(L, R) \propto L / R$. Without going into the details of the physical mechanism that makes the blocks move, the model assumes that each block moves randomly by $\Delta(R)$, with zero mean and the rms value of the displacement being proportional to $\left[\left\langle\Delta^{2}(R)\right\rangle\right]^{1 / 2} \propto R^{\lambda}$, where $\lambda$ is a parameter. Over any given time interval $T$, the number of the jumps $N_{j}(T, R)$ for various block sizes scales as $N_{j}(T, R) \propto T / R^{\mu}$, where $\mu$ is another parameter.

In computer simulations, each block was considered as a two-dimensional square; the sizes of blocks had been chosen to be $R=1,2,4,8,16, \ldots, 2048$. The displacement of each the 4096 surface points of the surface is determined as the sum of the displacements of blocks located just beneath it. At each time step the blocks having the smallest

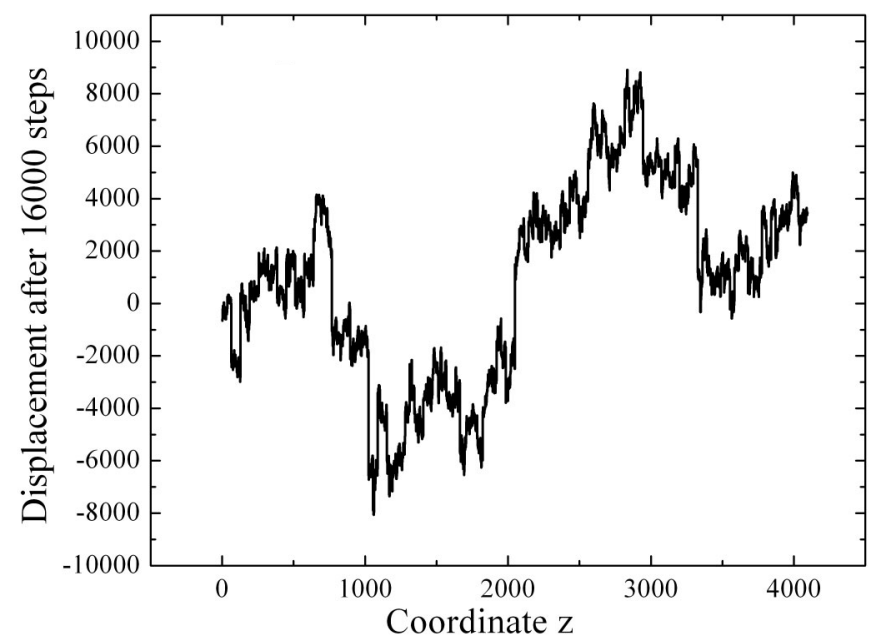

FIG. 45. Elevations of the set points after 16000 steps with parameters of the model $\lambda=\mu=1$. Coordinate $z$ is given in the units of the minimal block size.

dimension $R=1$ are randomly moved (vertically) with the displacement rms value equal to 1 ; blocks having $R=2$ are displaced after $2^{\mu}$ time steps randomly with rms value of the rms displacement equal to $2^{\lambda}$, etc. Figure 45 shows an example of the resulting profile after 16000 steps with the parameters of the model $\lambda=\mu=1$.

Figure 46 shows the dependence of the variance of the displacement $\left\langle d Y^{2}(t, L)\right\rangle$ on the distance between points for various time intervals $t=128,1024,4096$, and 32000 steps. One can see that, after 128 steps, the variance at the distances $L>128$ does not depend on $L$, i.e. $\left\langle d Y^{2}(t, L)\right\rangle \approx$ const The same phenomena occurs after 1024 steps at the distances $L>1024$. Assuming that all the moves are uncorrelated, the average variance of relative position changes for time intervals $T$ can be estimated as

$$
\begin{aligned}
\sigma^{2}(T, L) & =\left\langle[Y(t+T, z+L)-Y(t+T, z)-Y(t, z+L)+Y(t, z)]^{2}\right\rangle \\
& =\left(\sum_{\text {blocks }}^{R_{\max }<L} \sum_{\text {jumps }}^{\text {over } T} \Delta(R)\right)^{2} \propto \sum_{\text {blocks }}^{R_{\max }<L} R^{2 \lambda}\left(\frac{T}{R^{\mu}}\right)=\sum_{\text {size } R=1}^{L}\left(\frac{L}{R}\right) R^{2 \lambda}\left(\frac{T}{R^{\mu}}\right)=T L \sum_{R=1,2,4,8, \ldots}^{L} R^{2 \lambda-1-\mu} .
\end{aligned}
$$

If the parameter $D=2 \lambda-1-\mu \leq 0$, the sum can be easily calculated and it scales as $\sigma^{2}(T, L) \propto T L$. If $D>0$, the summation yields $\sigma^{2}(T, L) \propto T L^{2 \lambda-\mu}$. Figure 47 illustrates how the variance $\sigma^{2}(T, L)$ scales with $L$ depending on the exponents $\alpha$ and $\beta$. Note, that the time dependence of the variance can be made different from $\propto T^{1}$ if the jump frequency scales with time nonlinearly $N_{j}(T, R) \propto T^{\nu} / R^{\mu}$. In general, one can conclude that dynamic fractal models like the one we just considered result in the space-time diffusive motion which is qualitatively similar to the one observed in the experimental data discussed in previous sections.

We should note here that the widely accepted Langevintype stochastic equation for the geological landscape evo- lution always consider, besides smoothing diffusion and erosion terms, an external stochastic noise source uncorrelated in both space and time and with finite variance-see, e.g., Ref. [54] for detailed review and discussion. Of course, under these assumptions, the resulted variance scales $\sigma^{2}(T, L) \propto T$ in the case of no smoothing and no erosion, leaving off any dependence on the distance between the observation points. We believe that such an ansatz is basically incorrect as the ground motion noise clearly shows its nonstationary character, certain correlation, and scaling laws both in space and in time. Besides the ATL-law observations, the fractal statistics of earthquakes [55] repudiates the notion of stationary uncorrelated noise as the source of the observed ground motion. 


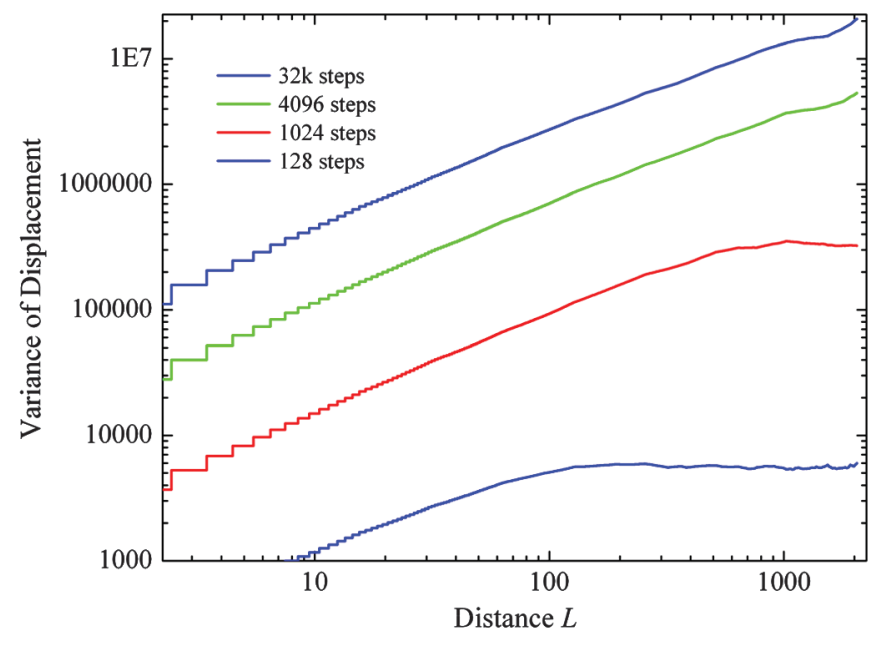

FIG. 46. (Color) Variance of the displacements vs distance between points for 128, 1024, 4096, and 32000 steps.

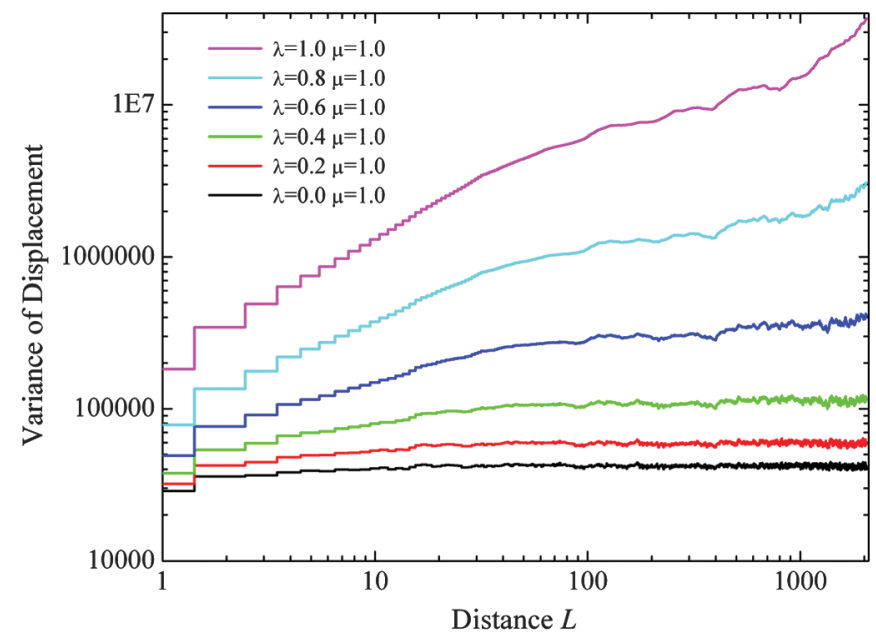

FIG. 47. (Color) Variance of the displacements vs distance between points after 16000 steps for different scaling exponents $\lambda$ and $\mu$ (see text).

\section{SUMMARY}

Numerous observations and analysis of the data on slow ground motion presented above reveal the phenomena of simultaneous ground diffusion in space and in time. The diffusion obeys a characteristic fractal law with the ground displacement variance $d Y^{2}$ scaling with time and spatial intervals $T$ and $L$ as $d Y^{2} \propto T^{\alpha} L^{\gamma}$ with both exponents close to $1(\alpha \approx \gamma \approx 1)$. The most suitable instruments for studying such diffusion are arrays of high precision instruments, e.g., hydrostatic level sensors connected by common water pipe and spread over significant area or regular laser tracking of numerous alignment monuments installed in large underground facilities like high energy accelerators. Nonrandom, systematic movements do often dominate the ground motion but the diffusion components still can be clearly identified using filtering methods. We believe that present landscape evolution models which assume random stochastic uncorrelated noise as a source of the ground motion are, therefore, incomplete. Our analysis shows that in many cases the data on the stochastic ground motion can be approximated by a simple empirical formula $\left\langle d Y^{2}\right\rangle=$ ATL which allows to estimate the longterm movements of accelerator tunnels and components and take them into account in the design simulations of accelerator facilities as long as the site dependent diffusion constant $A$ is determined.

\section{ACKNOWLEDGMENTS}

The author acknowledges very fruitful long-term collaboration on the development of HLS probes suitable for the ground motion studies for large accelerators and series of the studies with them at various places within the FNAL-SLAC-BINP team (Batavia-Stanford-Novosibirsk) of B. Baklakov, A. Chupyra, A. Erokhin, J. Lach, A. Medvedko, M. Kondaurov, V. Parkhomchuk, S. Singatulin, A. Seryi, E. Shubin, and J. Volk. I am very thankful to the collaborators who provided me with numerous records of raw data for further ground diffusion analysis-Professor S. Takeda, Dr. N. Yamomoto, Professor K. Oide, and Dr. M. Masuzawa (KEK, Japan), Dr. F. Tecker, Dr. J.-P. Quesnel, and Dr. M. Mayoud (CERN), the Fermilab's Alignment Group. Over the years I have had the pleasure of collaborating on theoretical studies of the ground motion effects on high energy particle accelerators with Dr. G. Stupakov, Dr. A. Seryi, and Dr. T. Raubenheimer (SLAC), Dr. R. Steining (SSCL), Dr. V. Lebedev (FNAL), Dr. J. Rossbach (DESY), and Dr. C. Montag (BNL). My special acknowledgements to Professor Vasily Parkhomchuk of Budker INP (Novosibirsk, Russia) who-at the time when we both were working on the design of a linear $e^{+} e^{-}$collider VLEPP — brought my attention to deep underlying physics issues associated with ground motion. He also was the first who coined the term "ATL law" while trying to analyze results of long-term measurement of the alignment monuments motion at the site of the UNK collider (Protvino, Russia). I am indebted to R. Carrigan for numerous suggestions which helped to improve the manuscript. Fermilab is operated by Fermi Research Alliance, LLC under Contract No. DE-AC02-07CH11359 with the United States Department of Energy.

[1] Gravitational Waves, edited by I. Ciufolini, V. Gorini, U. Moschella, and P. Fre (IOP Publishing, Philadelphia, 2001).

[2] The Detection of Gravitational Waves, edited by David G. Blair (Cambridge University Press, Cambridge, UK, 1991). 
[3] J. A. Giaime, E. J. Daw, M. Weitz, R. Adhikari, P. Fritschel, R. Abbott, R. Bork, and J. Heefner, Rev. Sci. Instrum. 74, 218 (2003).

[4] G. E. Fischer, in Summer School on High Energy Particle Accelerators, Batavia (1984), AIP Conf. Proc. No. 153 (AIP, New York, 1987), pp. 1047-1119.

[5] B. Baklakov, T. Bolshakov, A. Chupyra, A. Erokhin, P. Lebedev, V. Parkhomchuk, Sh. Singatulin, J. Lach, and V. Shiltsev, Phys. Rev. ST Accel. Beams 1, 031001 (1998).

[6] V. V. Parkhomchuk, V.D. Shiltsev, and G. V. Stupakov, Part. Accel. 46, 241 (1994).

[7] A. Sery and O. Napoly, Phys. Rev. E 53, 5323 (1996).

[8] See, e.g., series of Proceedings of the International Workshop on Accelerator Alignment [http://www-conf. slac.stanford.edu/iwaa/].

[9] D. C. Agnew, Rev. Geophys. 24, 579 (1986).

[10] J. Berger, in Advances in Geophysics, edited by H.E. Landsberg and J. van Mieghem (Academic Press, New York, 1973), Vol. 16.

[11] J. Havskov and G. Alguacil, Instrumentation in Earthquake Seismology (Springer, Dordrecht, Netherlands, 2004).

[12] B. D. Malamud and D. L. Turcotte, Adv. Geophys. 40, 1 (1999).

[13] Fractals in the Earth Sciences, edited by C. C. Barton and P. R. La Pointe (Plenum Press, New York, 1995).

[14] Fractals and Chaos in Geology and Geophysics, edited by D. L. Turcotte (Cambridge University Press, Cambridge, UK, 1997), 2nd ed.

[15] B. A. Baklakov, P. K. Lebedev, V. V. Parkhomchuk, A. A. Sery, A. I. Sleptsov, and V. D. Shiltsev, Tech. Phys. 38, 894 (1993); translated from Sov. Zh. Tech. Fiz. 63, 123 (1993).

[16] Handbook of Accelerator Physics and Engineering: A Compilation of Formulae \& Data, edited by A. Chao and M. Tigner (World Scientific, Singapore, 1999), Secs. 4.5 and 5.1.

[17] V. Shiltsev, in Proceedings of the International Workshop on Accelerator Alignment IWAA'95 (KEK Report No. KEK-Proceedings 95-12, Tsukuba, Japan, 1995), pp. 352-381; see also Ref. [8].

[18] R. Brinkmann and J. Rossbach, Nucl. Instrum. Methods Phys. Res., Sect. A 350, 8 (1994).

[19] H. Koiso, S. Kamada, and N. Yamamoto, Part. Accel. 27, 71 (1990).

[20] M. Masuzawa, H. Koiso, K. Oide, R. Sugahara, N. Yamamoto, and M. Yoshioka, in Proceedings of the International Workshop on Accelerator Alignment IWAA'2002 (Ref. [8]); the raw data files are kindly provided by $\mathrm{K}$. Oide.

[21] V. Shiltsev, T. Johnson, and X. L. Zhang, in Proceedings of the 26th Advanced ICFA Beam Dynamics Workshop on Nanometer Size Colliding Beams (Nanobeam-2002), Lausanne, (Switzerland) (CERN Proceedings-2003-001, 2003), pp. 97-103.

[22] F. Tecker, Report No. CERN-SL-96-40 BI 1996.

[23] R. Steinhagen, S. Redaelli, and J. Wenninger, Report No. CERN-AB-2005-087, 2005.

[24] V. Shiltsev, in Proceedings of the 22nd Advanced ICFA Beam Dynamics Workshop on Ground Motion in Future Accelerators, SLAC Stanford, 2000 (Report No. SLACWP-018, 2000), pp. 201-218.
[25] L. Arnaudon, B. Dehning, A. Hofmann, P. GrosseWiesmann, R. Jacobsen, J.P. Koutchouk, J. Miles, R. Olsen, M. Placidi, R. Schmidt, J. Wenninger, R. Assmann, A. Blondel, and G. E. Fischer, Nucl. Instrum. Methods Phys. Res., Sect. A 357, 249 (1995).

[26] M. Haubin, M. Mayoud, J.-P. Quesnel, and A. Verdier, CERN Report No. CERN-SL-94-44, 1994; the raw data files are kindly provided by J.-P. Quesnel.

[27] A. Seryi, in Proceedings of the 22nd Advanced ICFA Beam Dynamics Workshop on Ground Motion in Future Accelerators, SLAC Stanford, 2000 (Report No. SLACWP-018, 2000), pp. 297-302.

[28] V. Shiltsev and R. Stiening, SSCL-Preprint-505, 1993.

[29] V. Shiltsev, Phys. Rev. Lett. 104, 238501 (2010).

[30] V. Shiltsev, J. Volk, and S. Singatulin, in Proceedings of the 23rd Particle Accelerator Conference, Vancouver, Canada, 2009 (IEEE, Piscataway, NJ, 2009), TH5PFP020.

[31] A. Seryi, in Proceedings of the 22nd Advanced ICFA Beam Dynamics Workshop on Ground Motion in Future Accelerators, SLAC Stanford, 2000 (Report No. SLACWP-018, 2000), pp. 283-302.

[32] D. C. Agnew, Geophys. Res. Lett. 19, 333 (1992).

[33] W. B. Herrmannsfeldt, IEEE Trans. Nucl. Sci. 12, 9 (1965).

[34] C. Adolphsen, J. Irwin, G. Mozaheri, T. Slaton, and F. Zimmermann, in Proceedings of the 1997 Linear Collider Workshop, Protvino, Russia (Budker INP, Protvino, Russia, 1997)[http://www.desy.de/ conferences/LC97/proceed/html/097/01.htm].

[35] A. Seryi, Stanford Linear Accelerator Center Report No. SLAC-PUB-8597, 2000.

[36] J. Gervaise and E. J. N. Wilson, CERN Report No. CERN 87-01, 1987, pp. 128-182.

[37] V. Shiltsev, Proceedings of the 6th International Workshop on Linear Colliders (KEK Proceedings 95-5, Tsukuba, Japan, 1995).

[38] R. Assmann, C. Salsberg, and C. Montag, Stanford Linear Accelerator Center Report No. SLAC-PUB-7303, 1996.

[39] National Astronomical Observatory Mizusawa, in Report of The Coordinating Committee for Earthquake Prediction, edited by Geographical Survey Institute (Ministry of Construction, Japan, 1995), Vol. 53, p. 187.

[40] S. Takeda, A. Akiyama, K. Kudo, H. Nakanishi, and N. Yamomoto, in Proceedings of the 3rd International Workshop on Accelerator Alignment IWAA'93, Annecy, France; see Ref. [8].

[41] S. Takeda, H. Nakanishi, K. Kudo, and A. Akiyama, Int. J. Mod. Phys. A 2, 406 (1993).

[42] S. Takeda, K. Kudo, A. Akiyama, Y. Takeuchi, T. Katoh, Y. Kanazawa, and S. Suzuki, in Proceedings of the 5th European Particle Accelerator Conference (EPAC 96), Sitges, Spain (IOP Publishing, Philadelphia, 1996), pp. 2600-2602.

[43] S. Takeda, N. Yamamoto, M. Yoshioka, and Y. Nakayama, in Proceedings of the 10th European Particle Accelerator Conference, Edinburgh, Scotland, 2006 (EPS-AG, Edinburgh, Scotland, 2006), pp. 327-329.

[44] N. d'Oreye and W. Zuern, Rev. Sci. Instrum. 76, 024501 (2005); also in Proceedings of the International Workshop on Accelerator Alignment IWAA'2004, Geneva, Switzerland (Ref. [8]). 
[45] V. Shiltsev, J. Lach, B. Baklakov, A. Chupyra, A. Erokhin, M. Kondaurov, V. V. Parkhomchuk, E. Shubin, and S. Singatulin, in Proceedings of the Particle Accelerator Conference, Chicago, IL, 2001 (Ref. [48]), pp. 14701472.

[46] A. Seryi, R. Ruland, A. Chupyra, A. Erokhin, M. Kondaurov, A. Medvedko, V. Parkhomchuk, E. Shubin, S. Singatulin, J. Lach, D. Plant, V. Shiltsev, and A. Kuznetsov, in Proceedings of the 20th Particle Accelerator Conference, Portland, OR, 2003 (IEEE, New York, 2003), pp. 2769-2771.

[47] V. Shiltsev, Fermi National Accelerator Laboratory Report No. FERMILAB-FN-0717, 2002.

[48] A. Seryi, R. Ruland, B. Baklakov, A. Chupyra, A. Erokhin, M. Kondaurov, A. Medvedko, V. V. Parkhomchuk, E. Shubin, S. Singatulin, J. Lach, D. Plant, and V. Shiltsev, in Proceedings of the Particle Accelerator Conference, Chicago, IL, 2001 (IEEE, New York, 2001), pp. 1479-1481; see also A. Chupyra, M. Kondaurov, A. Medvedko, S. Singatulin, and E. Shubin, in Proceedings of the International Workshop on
Accelerator Alignment IWAA'2004, Geneva, Switzerland (Ref. [8]).

[49] E. Bonnet, O. Bour, N. E. Odling, P. Davy, I. Main, P. Cowie, and B. Berkowitz, Rev. Geophys. 39, 347 (2001).

[50] Monthly and Annual Mean Heights of Sea Level (Institute of Oceanographic Sciences, United Kingdom, 1978), Vol. 3.

[51] I. Rodríguez-Iturbe and A. Rinaldo Fractal River Basins: Chance and Self-Organization (Cambridge University Press, Cambridge, England, 2001); The Science of Fractal Images, edited by H. Peintgen and D. Saupe (Springer-Verlag, New York, 1988).

[52] A. Wolski and N. J. Walker, in Proceedings of the 20th Particle Accelerator Conference, Portland, OR, 2003 (Ref. [46]), pp. 2396-2398.

[53] V. Parkhomchuk and V. Shiltsev, Report No. Budker INP 92-31, 1992.

[54] P. S. Dodds and D. H. Rothman, Annu. Rev. Earth Planet Sci. 28, 571 (2000).

[55] P. Bak, K. Christensen, L. Danon, and T. Scanlon, Phys. Rev. Lett. 88, 178501 (2002). 